\title{
Holographic boundary states and dimensionally reduced braneworld spacetimes
}

\author{
Stefano Antonini॰* \\ Maryland Center for Fundamental Physics and Department of Physics, \\ University of Maryland, College Park, Maryland 20742, USA \\ Brian Swingle \\ Brandeis University, Waltham, Massachusetts 02453, USA and University of Maryland, \\ College Park, Maryland 20742, USA
}

(Received 17 May 2021; accepted 14 July 2021; published 24 August 2021)

\begin{abstract}
Recently it was proposed that microscopic models of braneworld cosmology could be realized in the context of anti-de Sitter/conformal field theory (AdS/CFT) correspondence using black hole microstates containing an end-of-the-world brane. Motivated by a desire to establish the microscopic existence of such microstates, which so far have been discussed primarily in bottom-up models, we have studied similar microstates in a simpler version of AdS/CFT. On one side, we define and study boundary states in the charged Sachdev-Ye-Kitaev model and show that these states typically look thermal with a certain pattern of symmetry breaking. On the other side, we study the dimensional reduction of microstates in EinsteinMaxwell theory featuring an end-of-the-world brane and show that they have an equivalent description in terms of 2D Jackiw-Teitelboim gravity coupled to an end-of-the-world particle. In particular, the same pattern of symmetry breaking is realized in both sides of the proposed duality. These results give significant evidence that such black hole microstates have a sensible microscopic realization.
\end{abstract}

DOI: 10.1103/PhysRevD.104.046023

\section{INTRODUCTION}

The anti-de Sitter/conformal field theory (AdS/CFT) correspondence [1-4] is one of the most promising frameworks for the formulation of a quantum theory of gravity. As a realization of the holographic principle [5,6], it relates the degrees of freedom of a quantum gravity theory in $d+1$ dimensions to those of a $d$-dimensional conformal field theory living on the conformal boundary of the spacetime manifold. However, while AdS/CFT provides a powerful microscopic description of certain classes of spacetimes, an important open question in quantum gravity is how to describe cosmological universes such as the phase of cosmic expansion we observe in the sky.

Inspired by the AdS/BCFT (boundary conformal field theory) proposal [7-9], one possible answer was provided by the holographic braneworld cosmology models recently proposed in $[10,11]$. These bottom-up models describe an asymptotically AdS black hole spacetime with the second

\footnotetext{
*santonin@umd.edu
}

Published by the American Physical Society under the terms of the Creative Commons Attribution 4.0 International license. Further distribution of this work must maintain attribution to the author(s) and the published article's title, journal citation, and DOI. Funded by SCOAP ${ }^{3}$. asymptotic region cut off by a spherically symmetric, constant tension, codimension-1 end-of-the-world (ETW) brane. The fields satisfy Neumann boundary conditions on the brane, which emerges from the past horizon and falls into the future horizon (in Lorentzian signature). From the point of view of a comoving observer, the radial motion of the brane is equivalent to the evolution of a closed FriedmannLemaitre-Robertson-Walker (FLRW) universe. When the brane is far from the black hole horizon, gravity is also localized on the brane [11] via a Randall-Sundrum mechanism [12-14], and the setup describes, to a good approximation, a cosmological model with expansion and contraction. In the Einstein-Maxwell model of [11], this only happened for large near-extremal charged black holes and near-critical branes. One possible microscopic realization of such models has been recently proposed by Van Raamsdonk in the context of $\mathcal{N}=4$ super-Yang-Mills (SYM) theory [15].

Similar black hole microstates have played an important role in recent discussions of the black hole information paradox [16,17]. They also provide toy models of the physics of nonunitary dynamics, which have recently received attention in the quantum statistical mechanics community in the context of quantum dynamics in the presence of random measurements [18-20]. Here the nonunitary dynamics is a Euclidean evolution starting at 
an initial state that can be thought of as the outcome of a random measurement. It is therefore of considerable interest to better understand the microscopic underpinnings of such black hole microstates. In particular, we would like to understand under what conditions various bottom-up constructions can be realized in a full microscopic model.

With the cosmological motivation foremost in our thoughts, the goal of the present paper is to use a special case of AdS/CFT duality to relate the near-horizon physics of the braneworld cosmology model proposed in [11], which involves a near-extremal AdS-Reissner Nordström (AdS-RN) black hole, with specific boundary states in the charged Sachdev-Ye-Kitaev (cSYK) model. In a similar fashion to the Majorana SYK construction by Kourkoulou and Maldacena [21], the boundary states considered are the fermionic Fock states, evolved for an amount $\tau_{0}$ of Euclidean time, called the preparation time. The boundary states can be prepared by a Euclidean path integral with a boundary condition at Euclidean time $\tau=-\tau_{0}[7,8,10,11,22,23]$. Here we continue to work with Einstein-Maxwell gravity as in [11], although the braneworld states we consider are in a different parameter regime than the one with good gravity localization (near the horizon versus far from the black hole, respectively). The matching we exhibit between braneworld spacetimes and microscopic boundary states is a step towards exhibiting a complete microscopic description of a cosmological spacetime.

We work in the context of the recent but already wellstudied example of AdS/CFT duality known as nearly $\mathrm{AdS}_{2} / \mathrm{CFT}_{1}$ duality [24-26]. The particular setup we consider involves Jackiw-Teitelboim (JT) gravity [27,28] and the low-energy limit of the Sachdev-Ye-Kitaev (SYK) model $[29,30]$. JT gravity is a $1+1$-dimensional dilatongravity theory whose saddle-point solution involves an $\mathrm{AdS}_{2}$ bulk spacetime with a dynamical boundary. The leading order fluctuations above the saddle point are governed by a boundary Schwarzian effective action [25]. JT gravity gives a good approximation to the physics near the horizon of a higher- dimensional, near-extremal charged black hole [25,26,31-35]. In particular, the Schwarzian fluctuations account for the deviation of the near-horizon geometry from $\mathrm{AdS}_{2} \times S^{d-1}$ [for a $(d+1)$ dimensional black hole]. For this reason, JT gravity is sometimes referred to as nearly $\mathrm{AdS}_{2}$ gravity.

The SYK model is a $(0+1)$-dimensional quantum mechanical model of $N$ interactive Majorana fermions. In the low-energy limit it develops an emergent conformal symmetry under arbitrary time diffeomorphisms $\operatorname{Diff}(\mathbb{R})$ [or $\operatorname{Diff}\left(S^{1}\right)$ in the thermal case]. Such symmetry is spontaneously broken down to $\operatorname{SL}(2, \mathbb{R})$ by the saddlepoint solution, which is exact in the large $N$ limit. This breaking generates an infinite number of degenerate zero modes. The symmetry is also explicitly broken down to $\mathrm{SL}(2, \mathbb{R})$ by a Schwarzian effective action at low but nonvanishing temperatures, which lifts the degeneracy of the zero modes. The residual $\operatorname{SL}(2, \mathbb{R})$ symmetry can be regarded as a gauge symmetry [24]. These features mimic exactly the properties of JT gravity [25], making the duality between low-energy SYK in the large $N$ limit and JT gravity manifest (at least near its saddle point; for a nonperturbative treatment see for example [36-39]). The SYK model can be generalized to higher dimensions [40] and to include continuous global symmetries [41-47]. A model of particular interest for our purposes is the SYK model with complex fermions (cSYK) [43-47], which possesses a global $U(1)$ symmetry. This is the correct model to describe the near-horizon physics of a dimensionally reduced near-extremal black hole. Indeed, unlike Majorana SYK, it can capture also charge fluctuations and describe charged bulk fermions $[43,44]$.

The main advantage of this model is that the duality can be realized explicitly in a simple setup. In particular, the cSYK boundary states can be constructed and studied. In analogy with the Majorana SYK case [21], we show that cSYK boundary states look thermal with inverse temperature $\beta=2 \tau_{0}$ and with properties analogous to those described by the canonical ensemble for a fixed-charge superselection sector (for a treatment of the nearly $\mathrm{AdS}_{2} / \mathrm{CFT}_{1}$ duality in the grand-canonical ensemble, including charge fluctuations, see for instance [31]). In particular, at large $N$ and when the preparation time is sufficiently large, the dynamics is governed by a Schwarzian effective action for the time reparametrization mode. Differently from the thermal case, the mode must satisfy appropriate boundary conditions, which are dictated by the total occupation number of the Fock state chosen (i.e., by the charge subsector considered). Such boundary conditions break two of the three generators of the residual $\operatorname{SL}(2, \mathbb{R})$ symmetry.

We also study the other side of the duality by performing a dimensional reduction of the braneworld cosmology model [11]. ${ }^{1}$ The near-horizon region is well approximated by JT gravity with a regularized boundary. We then show that configurations where the brane is tangent to the regularized boundary and the Schwarzian fluctuations of the boundary preserve such intersection point exhibit exactly the same pattern of symmetry breaking that we observe in the cSYK boundary states.

These results provide strong evidence for the duality between asymptotically AdS black holes containing an ETW brane and pure boundary states in a dual quantum mechanical theory, thereby realizing explicitly a simplified version of the holographic braneworld cosmology proposals $[10,11]$. However, we remark that this simple example is not directly relevant for the description of cosmology. Indeed, it describes a setup where the brane is in the nearhorizon region of a large AdS-RN black hole, and therefore

\footnotetext{
${ }^{1}$ Note that such model is also formulated in the canonical fixed-charge ensemble.
} 
gravity is not localized on the brane [11]. The holographic description of spacetimes with branes that are far from the black hole horizon and therefore can localize gravity likely requires more complicated boundary states of higherdimensional CFTs such as $\mathcal{N}=4$ SYM [15].

The paper is organized as follows. In Sec. II the relevant features of the cSYK model are quickly reviewed. In Sec. III the boundary states are defined in analogy with [21]. We study their properties, emphasizing their similarities with the thermal state using both analytic arguments and numerical evidence. Using the collective field formulation of the path integral, the Schwarzian effective action governing the dynamics of the time reparametrization mode is derived, and we find the two boundary conditions that the mode needs to satisfy, commenting on the resulting symmetry breaking pattern. In Sec. IV we briefly review the bottom-up holographic braneworld cosmology model proposed in [11]. Section V is devoted to the dimensional reduction of such model. The Schwarzian effective action is derived along with an equation of motion describing the trajectory of the brane. We show that when the brane is tangent to the regularized boundary and such property is preserved under Schwarzian fluctuations of the boundary, the system displays a symmetry-breaking pattern analogous to that found for the cSYK boundary states. In Sec. VI we discuss our findings and give final remarks. Our choice of units is $\hbar=c=\varepsilon_{0}=k_{B}=1$.

\section{REVIEW OF THE CSYK MODEL}

We consider the SYK model $[41,44,46,47]$ with $N$ complex fermions, with Hamiltonian

$$
\hat{H}=\sum_{\substack{j_{1}<\ldots<j_{q / 2}=1 \\ k_{1}<\ldots<k_{q / 2}=1}}^{N} J_{j_{1} \ldots j_{q / 2}, k_{1} \ldots k_{q / 2}} \mathcal{A}\left(\hat{\psi}_{j_{1}}^{\dagger} \ldots \hat{\psi}_{j_{q / 2}}^{\dagger} \hat{\psi}_{k_{1}} \ldots \hat{\psi}_{k_{q / 2}}\right),
$$

where $\mathcal{A}$ denotes the completely antisymmetrized product ${ }^{2}$ and the fermions satisfy the anticommutation relations $\left\{\hat{\psi}_{i}, \hat{\psi}_{j}^{\dagger}\right\}=\delta_{i j},\left\{\hat{\psi}_{i}, \hat{\psi}_{j}\right\}=0=\left\{\hat{\psi}_{i}^{\dagger}, \hat{\psi}_{j}^{\dagger}\right\}$. The couplings $J_{j_{1} j_{2}, k_{1} k_{2}}$ are independently drawn from a complex Gaussian distribution with zero mean and variance

$$
\overline{\mid J_{j_{1} \ldots j_{q / 2},\left.k_{1} \ldots k_{q / 2}\right|^{2}}}=J^{2} \frac{(q / 2) !(q / 2-1) !}{N^{q-1}}
$$

and satisfy the symmetry properties $J_{i j, k l}=-J_{j i, k l}=$ $-J_{i j, l k}=J_{k l, i j}^{*}$.

The cSYK model possesses a globally conserved $U(1)$ charge $\hat{Q}=\sum_{j} \mathcal{A}\left(\hat{\psi}_{j}^{\dagger} \hat{\psi}_{j}\right)=\sum_{j} \hat{\psi}_{j}^{\dagger} \hat{\psi}_{j}-\frac{N}{2} \hat{\mathbb{1}}$, with $\hat{\mathbb{1}}$ the identity matrix. Since $[\hat{Q}, \hat{H}]=0$, it is possible to find a

\footnotetext{
${ }^{2}$ The completely antisymmetric definition of the Hamiltonian implies a particle-hole symmetry [44], which is not important for our purposes but simplifies the analysis.
}

basis of energy eigenstates that diagonalizes the charge. The Hilbert space splits into superselection sectors labeled by their charge $\mathcal{Q}$. For even $N, \mathcal{Q}$ is an integer number satisfying $-N / 2<\mathcal{Q}<N / 2$. Each charge subsector admits a basis of energy eigenstates, and the ground state is in the $\mathcal{Q}=0$ subsector [42].

For $q=4$, every energy eigenstate with $\mathcal{Q} \neq 0$ is twofold degenerate due to the particle-hole symmetry: for every energy eigenstate in the $\mathcal{Q}=\overline{\mathcal{Q}}$ charge subsector, there is an energy eigenstate with the same energy eigenvalue in the $\mathcal{Q}=-\overline{\mathcal{Q}}$ charge subsector. Energy eigenstates with $\mathcal{Q}=0$ are nondegenerate.

The two-point function over a generic state $|\psi\rangle$ is defined as

$$
G\left(\tau_{1}, \tau_{2}\right)=-\frac{1}{N} \sum_{i=1}^{N}\left\langle\psi\left|T\left[\hat{\psi}_{i}\left(\tau_{1}\right) \hat{\psi}_{i}^{\dagger}\left(\tau_{2}\right)\right]\right| \psi\right\rangle,
$$

where $T$ is the fermionic time-ordered product, and therefore $G\left(\tau, \tau \pm 0^{+}\right)=\langle\hat{Q}\rangle / N \pm 1 / 2$, where $\langle\hat{Q}\rangle$ is the expectation value of the charge in the state $|\psi\rangle$. In a given charge subsector, this will be just the charge $\mathcal{Q}$ of the subsector. Note that the two-point function presents an UV asymmetry. The "off-diagonal" two-point correlators involving the product of two different fermions are suppressed by a power of $1 / N[24,40]$, as follows from a diagrammatic calculation of the variance.

In particular, we can consider a thermal state in the canonical ensemble at fixed charge. ${ }^{3}$ Then after averaging over the random couplings, the partition function is encoded in an effective action for two bilocal collective fields $(G, \Sigma)$ $[24,44]$. On shell, the $G$ field is the Green function defined in Eq. (3) with the state taken to be thermal, while $\Sigma$ is the selfenergy. The Euclidean action is given by

$$
\begin{aligned}
\frac{S_{E}}{N}= & -\log \operatorname{det}\left[\delta\left(\tau_{1}-\tau_{2}\right) \partial_{\tau_{1}}-\Sigma\left(\tau_{1}, \tau_{2}\right)\right] \\
& -\int d \tau_{1} d \tau_{2}\left[\Sigma\left(\tau_{1}, \tau_{2}\right) G\left(\tau_{2}, \tau_{1}\right)\right. \\
& \left.+\frac{J^{2}}{q}\left(-G\left(\tau_{1}, \tau_{2}\right) G\left(\tau_{2}, \tau_{1}\right)\right)^{\frac{q}{2}}\right] .
\end{aligned}
$$

Note that in the large $N$ limit the saddle-point solution fully determines the partition function with fluctuations suppressed by $1 / N$. In the IR, the first term in the determinant can be dropped and the action develops a symmetry under arbitrary time reparametrizations

\footnotetext{
${ }^{3}$ Since the braneworld cosmologies we consider are saddlepoint solutions that dominate in the gravitational canonical ensemble at fixed charge, the canonical partition function is the one relevant for our simple model. But in general charge fluctuations can be included in the analysis by considering the grandcanonical partition function for a fixed chemical potential $\mu$ [44].
} 
$\tau \rightarrow f(\tau)$, analogously to the Majorana SYK model. In the complex SYK case, there is an additional emergent local $U(1)$ symmetry. The IR saddle-point solutions for the collective fields transform conformally under time reparametrization. The general transformation law also includes a local phase shift:

$$
\begin{aligned}
G_{f, \lambda}\left(\tau_{1}, \tau_{2}\right) & =\left[f^{\prime}\left(\tau_{1}\right) f^{\prime}\left(\tau_{2}\right)\right]^{\Delta} e^{i\left[\lambda\left(\tau_{1}\right)-\lambda\left(\tau_{2}\right)\right]} G\left(f\left(\tau_{1}\right), f\left(\tau_{2}\right)\right), \\
\Sigma_{f, \lambda}\left(\tau_{1}, \tau_{2}\right) & =\left[f^{\prime}\left(\tau_{1}\right) f^{\prime}\left(\tau_{2}\right)\right]^{1-\Delta} e^{i\left[\lambda\left(\tau_{1}\right)-\lambda\left(\tau_{2}\right)\right]} \Sigma\left(f\left(\tau_{1}\right), f\left(\tau_{2}\right)\right) .
\end{aligned}
$$

The conformal IR (zero temperature) solution for the Green function $G$ is given by

$$
G_{\beta=\infty}( \pm \tau)=\mp \mathrm{e}^{ \pm \pi \mathcal{E}} b^{\Delta} \tau^{-2 \Delta}, \quad N \gg J \tau \gg 1,
$$

where $\tau=\tau_{1}-\tau_{2}, \beta$ is the inverse temperature and $\mathcal{E}$ is the asymmetry parameter, which is related to the average charge density [44]. The finite temperature solution in the $N \gg \beta J>\tau J \gg 1,(\beta-\tau) J \gg 1$ limit can be found by a time reparametrization $\tau \rightarrow \tan (\pi \tau / \beta)$ and an appropriate local phase shift $i \lambda(\tau)=-2 \pi \mathcal{E} \tau / \beta$ :

$$
G_{c}( \pm \tau)=\mp b^{\Delta} \frac{\mathrm{e}^{ \pm 2 \pi \mathcal{E}\left(\frac{1}{2}-\frac{\tau}{\beta}\right)}}{\left[\frac{\beta}{\pi} \sin \left(\frac{\pi \tau}{\beta}\right)\right]^{2 \Delta}}, \quad 0<\tau<\beta
$$

Note that this solution is correctly antiperiodic with period $\beta$.

The saddle-point solutions are not invariant under the transformation (5), meaning that both the symmetries are spontaneously broken. In particular, the $\operatorname{Diff}(\mathbb{R})$ [or $\operatorname{Diff}\left(S^{1}\right)$ at finite temperature] time reparametrization symmetry is spontaneously broken down to $\operatorname{SL}(2, \mathbb{R}),{ }^{4}$ and the local $U(1)$ symmetry is completely broken to global $U(1)$, which is an exact symmetry of the theory (see Appendix A and [45] for additional details).

The symmetries are also explicitly broken when including corrections away from the IR action. This means that, given the true saddle-point solution $\left(G_{*}, \Sigma_{*}\right)$, the solutions obtained by the transformation (5) have now an action which is lifted by a term of order $1 /(\beta J)$. They are "quasisolutions" that dominate the fluctuations around the saddle point at leading order in the temperature. Such dynamics is encoded at large $N$ in a Schwarzian effective action for the time reparametrization mode and an additional action for the phase field $\lambda(\tau)$ [44], which is

\footnotetext{
${ }^{4}$ Considering only bosonic fields (like $G$ ), we are not able to tell whether the residual symmetry group is $\operatorname{SL}(2, \mathbb{R})$ or $\operatorname{PSL}(2, \mathbb{R})=\operatorname{SL}(2, \mathbb{R}) /\{ \pm \hat{\mathbb{1}}\}$. Since we are interested in general in a bulk theory that contains also fermions, we will consider the residual gauge group to be $\operatorname{SL}(2, \mathbb{R})$, which is the appropriate one for a theory with fermions [48]; see Appendix A.
}

conjugate to charge-density fluctuations in the grandcanonical ensemble [47].

The two modes can be decoupled by a change of variables:

$$
\lambda(\tau) \rightarrow \lambda(\tau)+i 2 \pi \mathcal{E} \frac{\tau-\phi(\tau)}{\beta} .
$$

In the fixed-charge ensemble we are interested in, the dynamics at low temperature and large $N$ is then governed only by the Schwarzian action for the reparametrization mode:

$$
I_{\text {eff }}[f]=-\frac{N \gamma}{4 \pi^{2}} \int_{0}^{\beta} d \tau\left\{\tan \left(\frac{\pi f(\tau)}{\beta}\right), \tau\right\}
$$

where now $f(\tau)$ is a monotonic function obeying $f(\tau+\beta)=f(\tau)+\beta, \gamma$ is the coefficient of the linear term of the specific heat at fixed charge, and $\{f(\tau), \tau\}$ is the Schwarzian derivative

$$
\{f(t), t\} \equiv \frac{f^{\prime \prime \prime}(t)}{f^{\prime}(t)}-\frac{3}{2}\left(\frac{f^{\prime \prime}(t)}{f^{\prime}(t)}\right)^{2} .
$$

The Schwarzian action is $\operatorname{SL}(2, \mathbb{R})$ invariant. Therefore, the diffeomorphism invariance is both spontaneously and explicitly broken down to $\operatorname{SL}(2, \mathbb{R})$. The residual symmetry must be regarded as an unphysical gauge symmetry [24]: modes that are $\operatorname{SL}(2, \mathbb{R})$ equivalent correspond to the same physical configuration and must be accounted for only once in the path integral. In other words, the path integral for the reparametrization mode is carried over modes $f(\tau)$ in the left quotient of $\operatorname{Diff}\left(S^{1}\right)$ by $\operatorname{SL}(2, \mathbb{R})$. We will show that when the path integral computes amplitudes in a given boundary state, the reparametrization mode must satisfy specific boundary conditions that further break the residual $\operatorname{SL}(2, \mathbb{R})$ symmetry, leaving only one unbroken generator. In the next section we will take $q=4$ in the Hamiltonian (1) for simplicity. Our results should however generalize immediately to any $q$ integer multiple of 4 . The generalization to other values of $q$ might present some differences due to the different spectral structure [42].

\section{CSYK BOUNDARY STATES AND SYMMETRY BREAKING}

\section{A. Definition of the boundary states}

Consider the Fock basis of the cSYK Hilbert space. A Jordan-Wigner transformation allows us to map the fermions $\hat{\psi}_{i}$ to spin operators. We can then denote a generic state of the Fock basis as

$$
\left|S_{i}\right\rangle=\left|\uparrow_{1} \downarrow_{2} \ldots \uparrow_{N-1} \uparrow_{N}\right\rangle .
$$

These states are evidently eigenstates of both the occupation number operator for a single fermion $\hat{n}_{k}=\hat{\psi}_{k}^{\dagger} \hat{\psi}_{k}$ with 
eigenvalue 0 or 1 , and of the total charge operator $\hat{Q}$ with eigenvalue $\mathcal{Q}=n_{\uparrow, \mathcal{Q}}-N / 2$, where $n_{\uparrow, \mathcal{Q}}$ is the total number of fermions in the $|\uparrow\rangle$ state. However they are clearly not energy eigenstates, and therefore their dynamics is nontrivial.

The Euclidean time evolution under the interaction Hamiltonian (1) maps any given Fock state $\left|S_{i}\right\rangle$ with charge $\mathcal{Q}$ to a superposition of all the Fock states in the same charge subsector, whose dimension is $D_{\mathcal{Q}}=$ $N ! /\left[n_{\uparrow, \mathcal{Q}} !\left(N-n_{\uparrow, \mathcal{Q}}\right) !\right]$. Since the Hamiltonian can be diagonalized in each charge subsector, we can write a given Fock state with charge $\mathcal{Q}$ as a linear superposition of all the energy eigenstates in the same charge subsector:

$$
\left|S_{i}\right\rangle=\sum_{k=1}^{D_{\mathcal{Q}}} c_{k}^{i}\left|E_{k}\right\rangle
$$

From now on, we will assume to be working within a charge subsector with charge $\mathcal{Q}$. The complex coefficients $c_{k}^{i}$ must satisfy the normalization condition $\sum_{k=1}^{D_{\mathcal{Q}}}\left|c_{k}^{i}\right|^{2}=1$. But given the structure of the Hamiltonian (1), we can say something more. As we reviewed in the previous section, the couplings $J_{j_{1} j_{2}, k_{1} k_{2}}$ governing the all-to-all interactions between the fermions are all drawn from the same distribution with zero mean and fixed $1 / N^{3}$-suppressed variance. Given an energy eigenstate $\left|E_{k}\right\rangle=\sum_{i=1}^{D_{\mathcal{Q}}} c_{i}^{k}\left|S_{i}\right\rangle$, we then expect the coefficients $c_{i}^{k}=\left(c_{k}^{i}\right)^{*}$ (where ${ }^{*}$ indicates the complex conjugate) weighting the contribution of each Fock state $\left|S_{i}\right\rangle$ in the superposition to be randomly distributed. In particular, we expect ${ }^{5}$ :

(i) Their phases to be uniformly distributed in $[-\pi, \pi]$;

(ii) After averaging over disorder, their squared norm to have mean $\overline{\left|c_{i}^{k}\right|^{2}}=1 / D_{\mathcal{Q}}$ and variance $\operatorname{var}\left(\left|c_{i}^{k}\right|^{2}\right)=a / D_{\mathcal{Q}}^{2}+\mathcal{O}\left(D_{\mathcal{Q}}^{-3}\right)$, where $a>1$ is an order $1 c$ number;

(iii) The covariance $\operatorname{cov}\left(\left|c_{i}^{k}\right|^{2},\left|c_{j}^{k}\right|^{2}\right)$ to be suppressed at least by a power $D_{\mathcal{Q}}^{-3}$. Note that it cannot vanish exactly due to the normalization constraint;

(iv) The correlation between the coefficients and the energy eigenvalues to be also strongly suppressed. We emphasize that whenever the charge per particle differs by a nonvanishing amount from $\pm 1 / 2$ at large $N$, the quantity $D_{\mathcal{Q}}$ is exponential in $N$ and much greater than $N$ at large $N$.

These assumptions are reasonable in part because the model enjoys an enhanced $U(N)$ symmetry at large $N$ arising from the suppressed variance of the random couplings. In particular, such symmetry permutes the various Fock states in a given charge subsector. Note that this is the analog of the emergent $O(N)$ symmetry in the $N$ fermion Majorana SYK model [21]. The presence of the

\footnotetext{
${ }^{5}$ Physical intuitions and numerical evidence motivating these claims are reported in Appendix B 1.
}

$U(N)$ symmetry simplifies the analysis of the physics in the boundary states, especially when considering collective operators (see Appendixes B 1 and B 3).

Given the relation (12) and the properties of the coefficients $c_{k}^{i}$ that we have described, we expect Fock states to have energy of order zero. Since the ground-state energy of the cSYK model satisfies $E_{0} \propto-N$ [44], these are relatively high-energy states in the middle of the spectrum (for a given charge). In order to study the properties of boundary states in the well-understood and analytically treatable IR limit of the cSYK model, we can define our boundary states by evolving Fock states for an amount $\tau_{0}$ of Euclidean time, called the preparation time:

$$
\left|B_{i}\right\rangle=\frac{\mathrm{e}^{-\tau_{0} \hat{H}}\left|S_{i}\right\rangle}{\sqrt{\left\langle S_{i}\left|\mathrm{e}^{-2 \tau_{0} \hat{H}}\right| S_{i}\right\rangle}}=\frac{\sum_{k=1}^{D_{\mathcal{Q}}} c_{k}^{i} \mathrm{e}^{-\tau_{0} E_{k}}\left|E_{k}\right\rangle}{\sqrt{\sum_{k=1}^{D_{\mathcal{Q}}}\left|c_{k}^{i}\right|^{2} \mathrm{e}^{-2 \tau_{0} E_{k}}}} .
$$

If $\tau_{0}$ is sufficiently large, higher-energy eigenstates in the superposition are strongly suppressed and $\left|B_{i}\right\rangle$ is a lowenergy state. We remark that, in the large $N$ limit we are interested in, $N$ is the largest parameter in the theory and the spacing between energy eigenvalues is exponentially suppressed in $N$. The typical level spacing at a given energy density and charge is of order $\mathrm{e}^{- \text {entropy }}$ which is $1 / D_{\mathcal{Q}}$ in the middle of the spectrum. Therefore, the dynamics of the boundary state $\left|B_{i}\right\rangle$ remains substantially different from the one of the ground state, even for large preparation times $\tau_{0}$ (provided $\tau_{0}$ does not scale with $N$ ).

\section{B. Single-fermion two-point function}

We can now study the behavior of the "diagonal" twopoint correlator of one specific fermion over a boundary state:

$$
\tilde{G}_{i, k}\left(\tau_{1}, \tau_{2}\right)=-\left\langle B_{i}\left|T\left[\hat{\psi}_{k}\left(\tau_{1}\right) \hat{\psi}_{k}^{\dagger}\left(\tau_{2}\right)\right]\right| B_{i}\right\rangle
$$

with $\tau_{1,2} \in\left[-\tau_{0}, \tau_{0}\right]$. Using the definition (13), this can be rewritten as

$$
\tilde{G}_{i, k}\left(\tau_{1}, \tau_{2}\right)=-\frac{\left\langle S_{i}\left|\mathrm{e}^{-\tau_{0} \hat{H}} T\left[\hat{\psi}_{k}\left(\tau_{1}\right) \hat{\psi}_{k}^{\dagger}\left(\tau_{2}\right)\right] \mathrm{e}^{-\tau_{0} \hat{H}}\right| S_{i}\right\rangle}{\left\langle S_{i}\left|\mathrm{e}^{-2 \tau_{0} \hat{H}}\right| S_{i}\right\rangle} .
$$

Let us analyze the denominator first. Since the Fock states $\left|S_{i}\right\rangle$ form a basis of the charge subsector, summing over all the Fock states in the subsector we obtain the partition function at fixed charge $\mathcal{Q}$ and temperature $\beta=2 \tau_{0}$ :

$$
\sum_{i=1}^{D_{\mathcal{Q}}}\left\langle S_{i}\left|\mathrm{e}^{-2 \tau_{0} \hat{H}}\right| S_{i}\right\rangle=\operatorname{Tr}_{\mathcal{Q}}\left(\mathrm{e}^{-2 \tau_{0} \hat{H}}\right)=Z_{\mathcal{Q}}\left[2 \tau_{0}\right] .
$$

But given the properties of the coefficients outlined in the previous section, after averaging over the couplings we expect the value of the denominator to be independent of the 


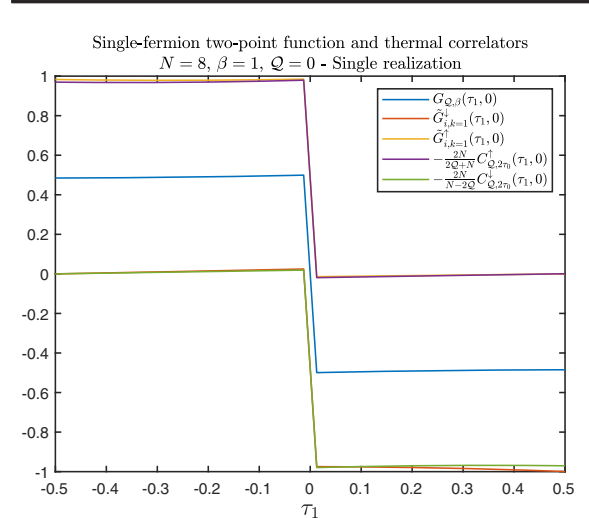

(a)

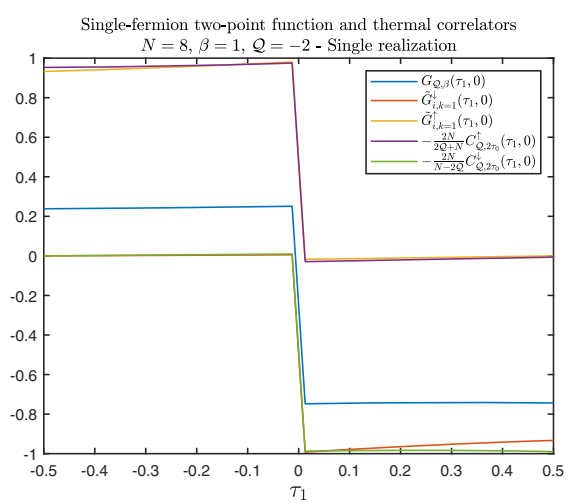

(b)

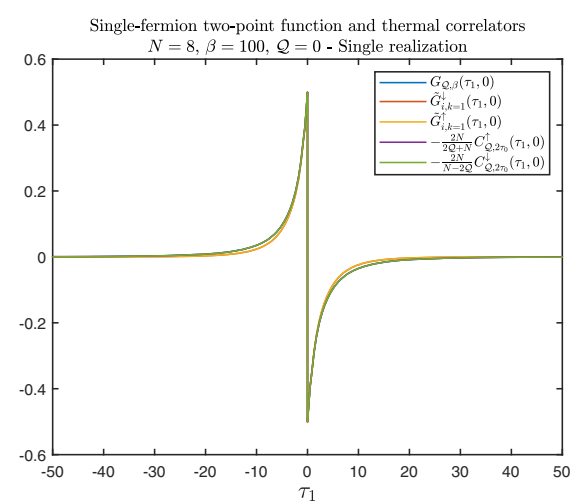

(c)

FIG. 1. Single-fermion two-point functions. The left-hand sides and right-hand sides of Eq. (18) are plotted along with the thermal two-point function in the corresponding charge subsector for $N=8, k=1$ and a single realization of the Hamiltonian. The expectations outlined in Sec. III B are matched to good accuracy. Note that for $\tau_{1} \approx \tau_{2}=0$ the thermal two-point function correctly takes the value $G\left(0^{ \pm}, 0\right) \approx \mathcal{Q} / N \mp 1 / 2$. (a) $\mathcal{Q}=0, \beta=2 \tau_{0}=1$. (b) $\mathcal{Q}=-2, \beta=2 \tau_{0}=1$. (c) $\mathcal{Q}=0, \beta=2 \tau_{0}=100$.

specific Fock state chosen. Additionally, since the distribution of the norms of the coefficients is peaked around their mean value, we expect this property to be approximately self-averaging. Therefore we get

$$
\left\langle S_{i}\left|\mathrm{e}^{-2 \tau_{0} \hat{H}}\right| S_{i}\right\rangle=\frac{1}{D_{\mathcal{Q}}} Z_{\mathcal{Q}}\left[2 \tau_{0}\right]+\mathcal{O}\left(D_{\mathcal{Q}}^{-1}\right) .
$$

Note that the first term is of order $D_{\mathcal{Q}}^{0}$. The analysis of the numerator of Eq. (15) is similar in fashion but slightly subtler, and is reported in Appendix B 2. We can expect that if one of the two fermion operators is inserted at time $\tau \approx \pm \tau_{0}$, or if the preparation time $\tau_{0}$ is not long enough, the correlator appearing in the numerator is strongly dependent on the occupation number of the $k$ th fermion (but not on the occupation number of all the other fermions). Conversely, if the preparation time is long enough $\left(\tau_{0} J \gg 1\right)$ and the fermions are both inserted far from the Fock states $\left[\left(\tau_{0}-\left|\tau_{1,2}\right|\right) J \gg 1\right]$, the correlator is approximately independent of all the occupation numbers, up to corrections of order $\exp \left(-\left(\tau_{0}-\left|\tau_{1,2}\right|\right) J\right)$. A self-averaging property analogous to the one described for the denominator holds also for the numerator.

In general, the correlation function (15) takes the form (up to $1 / D_{\mathcal{Q}}$-suppressed corrections)

$$
\begin{aligned}
& \tilde{G}_{i, k}^{\uparrow}\left(\tau_{1}, \tau_{2}\right)=-\frac{2 N}{2 \mathcal{Q}+N} C_{\mathcal{Q}, 2 \tau_{0}, k}\left(\tau_{1},-\tau_{0} ;-\tau_{0}+0^{+}, \tau_{2}\right), \\
& \tilde{G}_{i, k}^{\downarrow}\left(\tau_{1}, \tau_{2}\right)=-\frac{2 N}{N-2 \mathcal{Q}} C_{\mathcal{Q}, 2 \tau_{0}, k}\left(\tau_{1},-\tau_{0}+0^{+} ;-\tau_{0}, \tau_{2}\right),
\end{aligned}
$$

where the superscripts $\uparrow$ and $\downarrow$ indicate whether the $k$ th fermion is in the $|\uparrow\rangle$ or $|\downarrow\rangle$ state in the initial Fock state chosen and $C_{\mathcal{Q}, \beta, k}\left(\tau_{1}, \tau_{2} ; \tau_{3}, \tau_{4}\right)$ is the thermal four-point function at inverse temperature $\beta$, defined as [49]

$$
\frac{1}{Z_{\mathcal{Q}}[\beta]} \operatorname{Tr}_{\mathcal{Q}}\left(\mathrm{e}^{-\beta \hat{H}} T\left[\hat{\psi}_{k}\left(\tau_{1}\right) \hat{\psi}_{k}\left(\tau_{2}\right) \hat{\psi}_{k}^{\dagger}\left(\tau_{3}\right) \hat{\psi}_{k}^{\dagger}\left(\tau_{4}\right)\right]\right) .
$$

The result (18) is valid at leading order in $1 / D_{\mathcal{Q}}$ for any value of $\tau_{0}, \tau_{1}$, and $\tau_{2}$.

The properties of the coefficients $c_{i}^{k}$ outlined in the previous subsection guarantee that the value of the thermal correlator (19) is independent of $k$ up to corrections suppressed by an inverse power of $D_{\mathcal{Q}}$. Therefore, we can substitute $C_{\mathcal{Q}, 2 \tau_{0}, k} \rightarrow C_{\mathcal{Q}, 2 \tau_{0}}$, where $C_{\mathcal{Q}, 2 \tau_{0}}$ is averaged over all the fermions. Applying Wick's theorem at finite temperature [49] to the four-point function, and in the limit $\tau_{0} J \gg 1,\left(\tau_{0}-\left|\tau_{1,2}\right|\right) J \gg 1$, we find

$$
\tilde{G}_{i, k}^{\uparrow}\left(\tau_{1}, \tau_{2}\right) \approx \tilde{G}_{i, k}^{\downarrow}\left(\tau_{1}, \tau_{2}\right) \approx G_{\mathcal{Q}, \beta}\left(\tau_{1}, \tau_{2}\right),
$$

where $G_{\mathcal{Q}, \beta}\left(\tau_{1}, \tau_{2}\right)$ is the thermal two-point function at temperature $\beta=2 \tau_{0}$ averaged over all the fermions [which reduces to Eq. (7) in the large $N$ and $\tau J \gg 1$ limit]. As expected, in the appropriate limit the two-point correlator on the boundary state is independent of the specific Fock state chosen. Note that this result holds for any fermion $k$. The exact diagonalization numerical results reported in Fig. 1 confirm the predicted behavior. ${ }^{6}$ Equation (20) suggests that if the preparation time $\tau_{0}$ is large and if we measure observables not involving the insertion of a single fermion near the original Fock state, the dynamics in a generic boundary state with charge $\mathcal{Q}$ can be well approximated using the canonical ensemble at fixed charge $\mathcal{Q}$ and temperature $\beta=2 \tau_{0}$. In the next section we will show how

\footnotetext{
${ }^{6}$ Note that for the $N=8$ case we consider in our exact diagonalization results, evolving for a large amount of Euclidean time [for instance in the $\beta=100$ case of Fig. 1(c)] means to effectively end up in the ground state of the corresponding charge subsector. Additional details on this point are reported in Appendix B 2.
} 


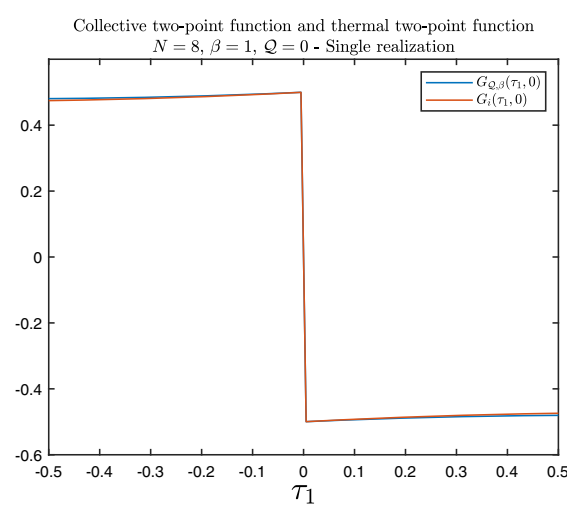

(a)

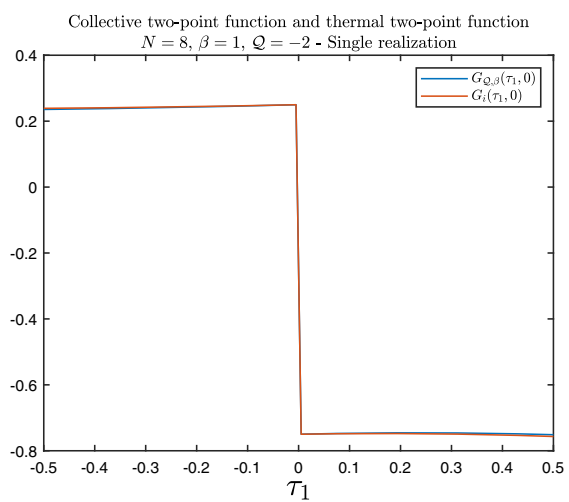

(b)

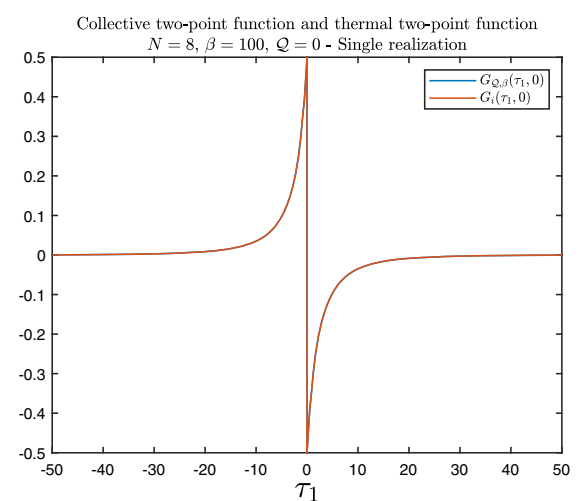

(c)

FIG. 2. Collective two-point functions. The left-hand side and right-hand side of Eq. (23) are plotted for $N=8, k=1$, and a single realization of the Hamiltonian. The expectations outlined in Sec. III C are matched to good accuracy. Note that for $\tau_{1} \approx \tau_{2}=0$ the collective and the thermal two-point functions correctly take the value $G\left(0^{ \pm}, 0\right) \approx \mathcal{Q} / N \mp 1 / 2$. (a) $\mathcal{Q}=0, \beta=2 \tau_{0}=1$. (b) $\mathcal{Q}=-2$, $\beta=2 \tau_{0}=1$. (c) $\mathcal{Q}=0, \beta=2 \tau_{0}=100$.

this property becomes even stronger by considering a collective two-point correlator, and in Sec. III D we will give a more general argument to link the dynamics in a boundary state to that one in the canonical ensemble.

\section{Collective two-point function}

We are ultimately interested in formulating the cSYK dynamics in a boundary state $\left|B_{i}\right\rangle$ in terms of the collective fields introduced in Sec. II. Indeed, in the large $N$ and large $\beta J$ limit, they are suitable to make the connection to the dual JT gravity picture. The first natural step towards a collective field formulation is to study the behavior of the collective two-point function over a boundary state:

$$
G_{i}\left(\tau_{1}, \tau_{2}\right)=-\left\langle B_{i}\left|T\left[\frac{1}{N} \sum_{k=1}^{N} \hat{\psi}_{k}\left(\tau_{1}\right) \hat{\psi}_{k}^{\dagger}\left(\tau_{2}\right)\right]\right| B_{i}\right\rangle .
$$

We have already pointed out that, up to corrections suppressed by powers of $1 / D_{\mathcal{Q}}$, the value of the single-fermion correlators is independent of the specific fermion $k$ chosen, as long as all the fermions considered have the same occupation number in the initial Fock state (or $\tau_{0} J \gg 1$ ). Therefore at large $N$ we can drop the subscript $k$ from the single-fermion correlator $\tilde{G}_{i}$. Since a Fock state with a given charge $\mathcal{Q}$ has $N / 2+\mathcal{Q}$ fermions in the $|\uparrow\rangle$ state and $N / 2-\mathcal{Q}$ fermions in the $|\downarrow\rangle$ state, the collective two-point function (21) then takes the form

$G_{i}\left(\tau_{1}, \tau_{2}\right) \approx\left(\frac{1}{2}+\frac{\mathcal{Q}}{N}\right) \tilde{G}_{i}^{\uparrow}\left(\tau_{1}, \tau_{2}\right)+\left(\frac{1}{2}-\frac{\mathcal{Q}}{N}\right) \tilde{G}_{i}^{\downarrow}\left(\tau_{1}, \tau_{2}\right)$.

Using Eq. (18) and Wick's theorem at finite temperature, we immediately obtain

$$
G_{i}\left(\tau_{1}, \tau_{2}\right) \approx G_{\mathcal{Q}, \beta}\left(\tau_{1}, \tau_{2}\right)
$$

for $\tau_{1,2} \in\left[-\tau_{0}, \tau_{0}\right]$. Note that, unlike the single-fermion correlator case, for the collective two-point function this result holds regardless of the value of the preparation time $\tau_{0}$ and of whether the fermions are inserted next to the Fock state. The numerical evidence reported in Fig. 2 confirms the result (23). When both fermions are inserted right next to the bra or the ket, the boundary value of the collective two-point functions is completely determined by its charge:

$$
\begin{aligned}
& G_{i}\left(-\tau_{0},-\tau_{0}^{-}\right)=G_{i}\left(\tau_{0}^{-}, \tau_{0}\right)=\frac{N+2 Q,}{2 N} \\
& G_{i}\left(-\tau_{0}^{-},-\tau_{0}\right)=G_{i}\left(\tau_{0}, \tau_{0}^{-}\right)=-\frac{N-2 Q,}{2 N}
\end{aligned}
$$

where $\tau_{0}^{-}=\tau_{0}-0^{+}$. In general, if the two fermions are inserted at the same time $\tau$, we obtain $G_{i}\left(\tau, \tau+0^{+}\right)=$ $(N+2 \mathcal{Q}) /(2 N)$ and $G_{i}\left(\tau+0^{+}, \tau\right)=-(N-2 \mathcal{Q}) /(2 N)$. When the two fermions are inserted one next to the bra and one next to the ket, at leading order the collective field takes the form

$\begin{aligned} G_{i}\left(-\tau_{0}, \tau_{0}\right) & =\frac{N+2 \mathcal{Q}}{2 N} \frac{D_{\mathcal{Q}}}{Z_{\mathcal{Q}}\left[2 \tau_{0}\right]} \frac{Z_{\mathcal{Q}-1}\left[2 \tau_{0}\right]}{D_{\mathcal{Q}-1}} \equiv C_{\mathcal{Q}}^{(1),} \\ G_{i}\left(\tau_{0},-\tau_{0}\right) & =-\frac{N-2 \mathcal{Q}}{2 N} \frac{D_{\mathcal{Q}}}{Z_{\mathcal{Q}}\left[2 \tau_{0}\right]} \frac{Z_{\mathcal{Q}+1}\left[2 \tau_{0}\right]}{D_{\mathcal{Q}+1}} \equiv C_{\mathcal{Q}}^{(2)} .\end{aligned}$

An important remark is in order here. Equations (23), (24), and (25) are independent of the specific boundary state $\left|B_{i}\right\rangle$. Therefore at large $N$ the collective two-point function provides information about the charge subsector, but is not able to distinguish between two boundary states within the same charge subsector. In principle, at leading order in $1 / N$ it also cannot distinguish a boundary state of 
the form described above from a boundary state obtained by evolving in Euclidean time a generic superposition of Fock states. Conversely, knowledge about the boundary value of all the single-fermion correlators introduced in the previous subsection is enough to identify the boundary state uniquely. The Schwarzian effective action will be derived in the collective field formulation. Therefore, the Schwarzian sector of our theory describes the physics in any boundary state in a given charge subsector, or in a boundary state built from a generic superposition of Fock states. This implies that the Schwarzian sector of our gravitational bulk theory will be dual to any boundary state of such class in a given charge subsector. From a bulk perspective, in order to tell apart two different boundary states in the same charge subsector we would need to take into account bulk fermions and provide a set of boundary conditions for them on the dimensionally reduced ETW brane. Note that $N$ such fields are naturally present in a bulk model dual to the low-energy limit of the cSYK model, and their limiting value on the regularized boundary represents the source for the cSYK fermions, according to the HKLL dictionary [50]. Additional remarks on this point will be made at the end of Sec. V. Although interesting, the question of how to implement such boundary conditions is beyond the goals of the present paper.

\section{Boundary states look thermal}

In this subsection we generalize the results obtained for two-point functions to generic operators $\hat{O}$. In particular, we show that in the large $N$ limit the expectation value of generic operators in a boundary state approaches the thermal expectation value computed by the canonical ensemble at inverse temperature $\beta=2 \tau_{0}$. We will make use of the following assumptions:

(1) The distribution of the coefficients $c_{i}^{k}$ satisfies the properties outlined in Sec. III A, i.e., the phases are uniformly distributed in $[-\pi, \pi]$, the norms of the coefficients satisfy $\overline{\left|c_{i}^{k}\right|^{2}}=1 / D_{\mathcal{Q}}, \quad \operatorname{var}\left(\left|c_{i}^{k}\right|^{2}\right)=$ $a / D_{\mathcal{Q}}^{2}+\mathcal{O}\left(D_{\mathcal{Q}}^{-3}\right), \operatorname{cov}\left(\left|c_{i}^{k}\right|^{2},\left|c_{j}^{k}\right|^{2}\right)=\mathcal{O}\left(D_{\mathcal{Q}}^{-3}\right)$, and the correlation between the coefficients and the energy eigenvalues is strongly suppressed.

(2) The matrix elements $\left\langle E_{i}|\hat{O}| E_{j}\right\rangle$ of the operator $\hat{O}$, which in general is a nonlocal operator in time, are weakly correlated with the coefficients $c_{i}^{k}$. This implies that the matrix elements $\left\langle B_{i}|\hat{O}| B_{i}\right\rangle$ depend weakly on the choice of the initial Fock state $\left|S_{i}\right\rangle$.

(3) The off-diagonal matrix elements are suppressed in the large $N$ limit: $\left\langle E_{i}|\hat{O}| E_{j}\right\rangle=\mathcal{O}\left(\mathrm{e}^{- \text {entropy } / 2}\right)$, which is $\mathcal{O}\left(1 / \sqrt{D_{\mathcal{Q}}}\right)$ in the center of the spectrum.

Assumption 1 has been already motivated, and is confirmed by the numerical results reported in Appendix B 1 . Assumption 2 holds up to $1 / N$ corrections for any operator $\hat{O}$ which is invariant under permutations of the fermions, or is inserted far from the Fock state at $\tau= \pm \tau_{0}$. This excludes operators involving the insertion of single fermions at time $\pm \tau_{0}$. In fact, that would cause $\left\langle B_{i}|\hat{O}| B_{i}\right\rangle$ to vanish identically for some boundary states. Such operators can clearly distinguish a boundary state from the thermal state (see Appendix B 3 for further discussion on this point). Assumption 3 is a standard form of the eigenstate thermalization hypothesis (ETH) [51-53].

Let us now study the behavior of the expectation value of an operator $\hat{O}$ over a boundary state with charge $\mathcal{Q}$ : $\langle\hat{O}\rangle_{i} \equiv\left\langle B_{i}|\hat{O}| B_{i}\right\rangle$. We outline here the reasoning and results of our analysis, while the details of the proof are reported in Appendix B 3. All the results are true in the large $N$ limit at leading order in $1 / D_{\mathcal{Q}}$. Using the definition of the boundary states (13), the average over disorder of the expectation value $\langle\hat{O}\rangle_{i}$ is equal to the expectation value of the operator $\hat{O}$ in the canonical ensemble at fixed charge $\mathcal{Q}$ and inverse temperature $\beta=2 \tau_{0}$ :

$$
\overline{\overline{\langle O\rangle_{i}}}=\frac{1}{Z_{\mathcal{Q}}\left[2 \tau_{0}\right]} \operatorname{Tr}_{\mathcal{Q}}\left(\mathrm{e}^{-2 \tau_{0} \hat{H}} \hat{O}\right)+\mathcal{O}\left(\frac{1}{D_{\mathcal{Q}}}\right)
$$

where we used a double overline to indicate that we averaged over both the phases and the norms of the coefficients $c_{k}^{i}$ (see Appendix B 3). Therefore, after averaging over the random couplings, every boundary state $\left|B_{i}\right\rangle$ "looks" thermal in the large $N$ limit. It is then natural to ask to what extent this property remains valid for a single realization of the Hamiltonian (1). The answer to this question can be found in the size of the fluctuations around the average value (26):

$$
\overline{\overline{\langle O\rangle_{i}^{2}}}-{\overline{\overline{\langle O\rangle_{i}}}}^{2}=\mathcal{O}\left(\frac{1}{D_{\mathcal{Q}}}\right)
$$

Equation (27) shows that fluctuations around the average value (26) are suppressed in the large $N$ limit. This implies that even for a single realization of the Hamiltonian the expectation value of a generic operator $\hat{O}$ over a boundary state approaches the thermal expectation value computed in the canonical ensemble, up to $1 / N$-suppressed corrections.

The takeaway is clear: unless measurements of very specific operators involving the insertion of single fermions at time $\tau \approx \pm \tau_{0}$ are performed, at leading order in the $1 / N$ expansion it is impossible to discriminate between a boundary state $\left|B_{i}\right\rangle$ and the thermal state. In other words, boundary states look thermal.

\section{E. Path integral, Schwarzian action, and symmetry breaking}

Euclidean correlators in a boundary state $\left|B_{i}\right\rangle$ can be computed using the Euclidean path integral with boundary conditions at Euclidean time $\tau= \pm \tau_{0}$ : 


$$
\left\langle B_{i}|\hat{O}| B_{i}\right\rangle=\int_{\left|\phi\left( \pm \tau_{0}\right)\right\rangle=\left|S_{i}\right\rangle} \mathcal{D} \psi \mathcal{D} \bar{\psi} O \mathrm{e}^{-S_{S Y K}[\psi, \bar{\psi}]},
$$

where we denoted with $\bar{\psi}$ the Grassmann variables associated with the fermion creation operators $\psi^{\dagger}$. The boundary condition $\left.\left|\phi\left( \pm \tau_{0}\right)=\right| S_{i}\right\rangle$ means that the occupation number of each fermion at time $\tau= \pm \tau_{0}$ is fixed.

It is then possible to average over the random couplings, introduce the collective fields $(G, \Sigma)$, and integrate out the fermions in complete analogy with the usual collective field formulation of the SYK model we reviewed in Sec. II. We arrive then at a path integral over the collective fields, where the action is given by Eq. (4) and now the boundary conditions $\left|\phi\left( \pm \tau_{0}\right)\right\rangle=\left|S_{i}\right\rangle$ become a set of boundary conditions on the collective field $G$, which is completely analogous to the one in Eqs. (24) and (25).

When the preparation time is large, $\tau_{0} J \gg 1$, all the IR analysis carried out in Sec. II is still valid. In particular, the action develops an emergent time reparametrization symmetry which is spontaneously and explicitly broken down to $\operatorname{SL}(2, \mathbb{R})$. The leading-order fluctuations around the saddle point are still dominated at large $N$ by Schwarzian fluctuations of the reparametrization mode, governed by the effective action (9) with $\beta=2 \tau_{0}$. Under time reparametrization $\tau \rightarrow f(\tau)$ [with $f(\tau)$ monotonic] the $(G, \Sigma)$ fields transform according to Eq. (5), where we can ignore the phase field ${ }^{7}$ after decoupling it from the reparametrization mode using the transformation (8).

There are two differences from the thermal case. The first one is that now the collective fields are defined only for $\tau_{1,2} \in\left[-\tau_{0}, \tau_{0}\right]$. Therefore, given the transformation law (5), every time diffeomorphism $\tau \rightarrow f(\tau)$ should map the interval $\left[-\tau_{0}, \tau_{0}\right]$ to itself. The second one is that the collective field $G$ must satisfy the boundary conditions (24) and (25). Given the true saddle-point solution $G_{*}$, which satisfies by definition Eqs. (24) and (25), we can use the transformation law (5) to derive two boundary conditions for the time reparametrization mode $f(\tau)$. Time reparametrizations satisfying such boundary conditions will map the true saddle to quasisolutions $G_{f}$ that also satisfy Eqs. (24) and (25).

We will use only the first lines of Eqs. (24) and (25). It is immediate to check that the boundary conditions for the reparametrization mode we find guarantee that the second lines of Eqs. (24) and (25) are satisfied as well. First, we can impose the boundary condition

$\frac{N+2 \mathcal{Q}}{2 N}=G_{f}\left(\tau_{0}^{-}, \tau_{0}\right)=\left[f^{\prime}\left(\tau_{0}\right)\right]^{2 \Delta} G_{*}\left(f\left(\tau_{0}^{-}\right), f\left(\tau_{0}\right)\right)$.

Using the monotonicity of $f(\tau)$ and the fact that the true saddle satisfies $G_{*}\left(\tau, \tau+0^{+}\right)=(N+2 \mathcal{Q}) /(2 N)$ for any

\footnotetext{
${ }^{7} \mathrm{We}$ ignore the phase field because it is conjugate to chargedensity fluctuations in the grand-canonical ensemble, and we are working at fixed charge $\mathcal{Q}$.
}

$\tau \in\left[-\tau_{0}, \tau_{0}\right]$, we get $G_{*}\left(f\left(\tau_{0}^{-}\right), f\left(\tau_{0}\right)\right)=(N+2 \mathcal{Q}) /(2 N)$ and therefore $f^{\prime}\left(\tau_{0}\right)=1$. Repeating the same argument for $G_{f}\left(-\tau_{0},-\tau_{0}^{-}\right)$we also obtain $f^{\prime}\left(-\tau_{0}\right)=1$. Let us now impose the third boundary condition:

$C_{\mathcal{Q}}^{(1)}=G_{f}\left(-\tau_{0}, \tau_{0}\right)=\left[f^{\prime}\left(-\tau_{0}\right) f^{\prime}\left(\tau_{0}\right)\right]^{\Delta} G_{*}\left(f\left(-\tau_{0}\right), f\left(\tau_{0}\right)\right)$.

Using the first boundary condition $f^{\prime}\left( \pm \tau_{0}\right)=1$, this reduces to $G_{*}\left(f\left(-\tau_{0}\right), f\left(\tau_{0}\right)\right)=C_{\mathcal{Q}}^{(1)}$. Since $f\left(\tau_{0}\right) \neq$ $f\left(-\tau_{0}\right)$ and we know that $G_{*}\left(-\tau_{0}, \tau_{0}\right)=C_{\mathcal{Q}}^{(1)}$, the only way to satisfy this condition is to impose $f\left( \pm \tau_{0}\right)= \pm \tau_{0}$. Therefore we arrived at a set of boundary conditions for the time reparametrization mode:

$$
f\left( \pm \tau_{0}\right)= \pm \tau_{0}, \quad f^{\prime}\left( \pm \tau_{0}\right)=1
$$

The same result was achieved by Kourkoulou and Maldacena in the context of the Majorana SYK model.

Note that, given a time diffeomorphism $f(\tau)$ that does not satisfy the boundary conditions (31), it is always possible to perform a $\operatorname{SL}(2, \mathbb{R})$ transformation to a $\tilde{f}(\tau)$ that satisfies (31) (see Appendix A). In Sec. II we emphasized that $\operatorname{SL}(2, \mathbb{R})$-equivalent modes must be accounted for only once in the path integral. Therefore, the boundary conditions (31) do not exclude any reparametrization mode from the path integral. They rather further break two out of the three generators of the residual $\operatorname{SL}(2, \mathbb{R})$ symmetry. Note that the same pattern of symmetry breaking is obtained, as we have pointed out, by starting with a generic superposition of Fock states instead of a single one.

A simple calculation yields the only unbroken generator. ${ }^{8}$ Consider a $\operatorname{SL}(2, \mathbb{R})$ transformation mapping the circle of length $2 \tau_{0}$ to itself:

$$
\mathrm{e}^{i \frac{\pi}{\tau_{0}} f(\tau)}=\mathrm{e}^{i \theta} \frac{\mathrm{e}^{i \frac{\pi}{\tau_{0}} \tau}+\alpha}{\alpha^{*} \mathrm{e}^{i \frac{\pi}{\tau_{0}} \tau}+1}, \quad|\alpha| \leq 1,
$$

where ${ }^{*}$ denotes the complex conjugate, $\alpha$ is a complex number, and $\theta \in[0,2 \pi]$. The infinitesimal transformation $(|\alpha| \ll 1)$ is

$$
f(\tau) \approx \tau+a+b \cos \left(\frac{\pi \tau}{\tau_{0}}\right)+c \sin \left(\frac{\pi \tau}{\tau_{0}}\right),
$$

with $a=\tau_{0} \theta / \pi, b=2 \tau_{0} \operatorname{Im}(\alpha) / \pi, c=-2 \tau_{0} \operatorname{Re}(\alpha) / \pi$. The three generators of the transformation are then $\left(1, \cos \left(\pi \tau / \tau_{0}\right), \sin \left(\pi \tau / \tau_{0}\right)\right)$. Imposing the boundary conditions (31) in Eq. (33) we find $a=b, c=0$ : the only $\mathrm{SL}(2, \mathbb{R})$ generator unbroken by the boundary conditions is

\footnotetext{
${ }^{8}$ We thank Rodrigo A. Silva for pointing this out.
} 


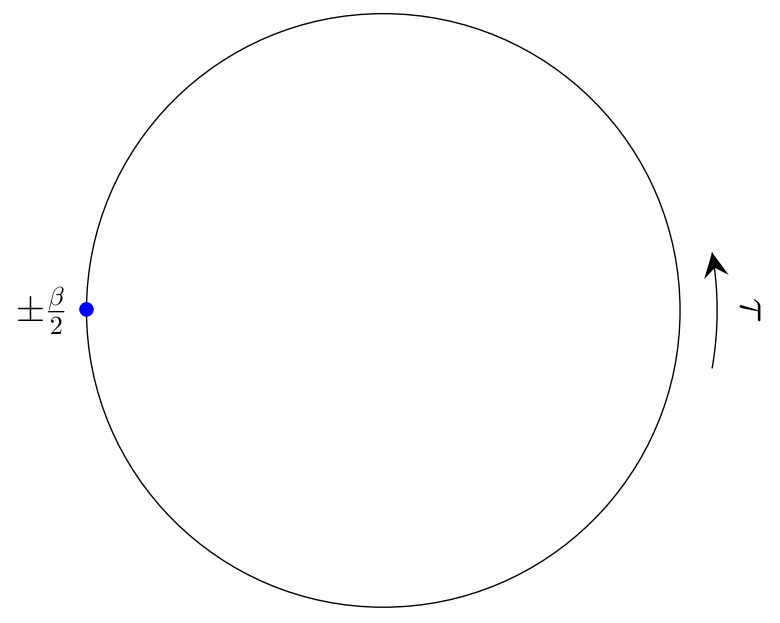

FIG. 3. Boundary states and canonical ensemble. The symmetry-breaking pattern we encountered when evaluating correlators over a boundary state can be visualized by considering the thermal circle with length $\beta=2 \tau_{0}$ with a fixed, special point at $\tau= \pm \tau_{0}$. The physics in a boundary state will then be equivalent to the one described by the canonical ensemble at fixed charge in the same charge subsector, except the collective fields must satisfy the appropriate boundary conditions at the special point.

the combination $1+\cos \left(\pi \tau / \tau_{0}\right)$. Note that this generator correctly maps the points $\pm \tau_{0}$ to themselves.

Finally, an intuitive picture that will be useful to guide us in the analysis of the gravity dual is that the physics in a boundary state $\left|B_{i}\right\rangle$ is analogous to the physics in the thermal state, but we have a fixed, special point on the thermal circle (which has length $\beta=2 \tau_{0}$ ) at the identified Euclidean times $\tau= \pm \tau_{0}$, where the boundary conditions for the collective fields in the path integral must be imposed (see Fig. 3).

\section{REVIEW OF HOLOGRAPHIC BRANEWORLD COSMOLOGIES}

In this section we briefly review the main features of the bottom-up holographic braneworld cosmology model built in [11]. The model is a generalization to the AdS-RN case of the AdS-Schwarzschild model originally proposed in [10].

The full $(d+1)$-dimensional gravitational Euclidean action (reported in Appendix C) is given by $S_{g}=S_{\text {bulk }}+$ $S_{\text {ETW }} \cdot S_{\text {bulk }}$ is the Einstein-Maxwell action with a negative cosmological constant, and includes a Gibbons-HawkingYork (GHY) term and an electromagnetic boundary term for the asymptotic boundary, needed when considering the gravitational ensemble at fixed charge $[54,55] . S_{\mathrm{ETW}}$ is the $d$-dimensional action for the constant tension ETW brane, also involving an electromagnetic boundary term. According to the AdS/BCFT prescription [7,8], all the bulk fields satisfy Neumann boundary conditions on the brane, which possesses a dynamical metric and cuts off the Euclidean geometry (see Fig. 4).
The saddle-point solution of the bulk action is given by the Euclidean AdS-RN wormhole, whose metric is

$$
d s^{2}=F(r) d \tau^{2}+\frac{d r^{2}}{F(r)}+r^{2} d \Omega_{d-1}^{2}
$$

where $d \Omega_{d-1}^{2}$ is the line element of the (d-1)-dimensional unit sphere and

$$
F(r)=1+\frac{r^{2}}{L_{\mathrm{AdS}}^{2}}-\frac{2 \mu}{r^{d-2}}+\frac{Q^{2}}{r^{2(d-2)}}
$$

with $L_{\text {AdS }}$ AdS radius and $\mu$ and $Q$ mass and charge parameters of the black hole, respectively. The metric has two horizons: an outer event horizon at $r=r_{+}$, and an inner Cauchy horizon at $r=r_{-}$. Varying the brane action (or, equivalently, imposing Israel junction conditions) yields an equation of motion for the brane

$$
\frac{d r}{d \tau}= \pm \frac{F(r)}{T_{\mathrm{ETW}} r} \sqrt{F(r)-T_{\mathrm{ETW}}^{2} r^{2}},
$$

where $T_{\mathrm{ETW}} \in\left(0,1 / L_{\mathrm{AdS}}\right)$ is the tension of the brane and the \pm signs correspond to the expanding and contracting phases of the brane, which reaches its minimum radius $r=r_{0}$ at $\tau= \pm \beta / 2$, where $\beta$ is the inverse temperature of the black hole. The preparation time $\tau_{0}$ of the dual CFT state can then be computed in terms of bulk parameters by subtracting from the total Euclidean periodicity $\beta$ the total time $2 \Delta \tau$ needed for the brane to complete its trajectory from and back to the asymptotic boundary: $\tau_{0}=(\beta-2 \Delta \tau) / 2$.

The $\tau=0, \pm \beta / 2$ slice is taken as initial condition for the evolution in Lorentzian time. In other words, the state on the $\tau=0, \pm \beta / 2$ slice is prepared by the gravitational Euclidean path integral with appropriate boundary conditions at $\tau=-\tau_{0}$, and the resulting state is further evolved in Lorentzian time. The corresponding Lorentzian geometry is given by the maximally extended AdS-RN black hole, where the left asymptotic region is cut off by the ETW brane. The minimum radius $r_{0}$ in Euclidean signature becomes a maximum radius in Lorentzian signature, and the brane emerges from the past event horizon in the left asymptotic region and collapses into the future event horizon. In principle, we can glue multiple patches of the AdS-RN spacetime, and extend farther the brane trajectory. It would then cross the inner horizon, reach a minimum value radius $r_{0}^{-}$, and then start expanding again, emerging in a new patch of the universe (see Fig. 5). However, the evolution of the brane trajectory after it crosses the Cauchy horizon is not reliable due to the instability of the latter [56]. Since gravity localization is also efficient only when the brane is far from the black hole horizon [11], we will focus our attention on the portion of trajectory that resides in the exterior region of the black hole. In this picture, the whole geometry is dual to a single BCFT with a $U(1)$ global symmetry living on the right 
asymptotic boundary. The Euclidean version of the CFT lives on $S^{d-1} \times\left[-\tau_{0}, \tau_{0}\right]$. From the point of view of an observer comoving with the brane, the Lorentzian trajectory of the brane in the black hole spacetime looks like the expansion and contraction of a closed FLRW universe, where the brane radius plays the role of the scale factor and satisfies the Friedmann equation:

$$
\left(\frac{\dot{r}}{r}\right)^{2}=-\frac{1}{r^{2}}+\frac{2 \mu}{r^{d}}-\frac{Q^{2}}{r^{2 d-2}}+\left(T_{\mathrm{ETW}}^{2}-\frac{1}{L_{\mathrm{AdS}}^{2}}\right),
$$

where $\dot{r}$ denotes a derivative with respect to the brane proper time. Although for $d=4$ the motion of the brane resembles the evolution of a cosmological universe, we obtain an effective four-dimensional braneworld cosmology only if an observer living on the brane perceives gravity as effectively four dimensional and localized on the brane. Gravity localization can be achieved via a RandallSundrum II mechanism [12,13] (see [14,57] for generalizations relevant to our discussion). In the presence of a bulk black hole, it is localized only locally and when the brane is far from the black hole horizon [11,57]. Therefore, in order to have a portion of the brane trajectory where gravity is localized, we need the maximum radius of the brane $r_{0}$ (i.e., the minimum radius in Euclidean signature) to be much larger than the black hole event horizon $r_{0} \gg r_{+}$. This can be obtained by considering a near-critical brane $\left(T_{\text {ETW }} \lesssim 1 / L_{\text {AdS }}\right)$ and a large black hole $\left(r_{+} \gg L_{\text {AdS }}\right)$.

In order for this gravitational model to describe a braneworld cosmology and have a BCFT dual, we need two conditions to be satisfied at the same time:

(1) The preparation time computed in terms of bulk parameters must be positive: $\tau_{0}>0$;

(2) For part of its trajectory the brane must sit far from the black hole horizon, i.e., $r_{0} \gg r_{+}$.

As we have already pointed out, condition 2 guarantees that gravity can be localized on the brane and that we can properly consider the evolution of the brane as the one of a cosmological universe. Condition 1 on the other hand guarantees the existence of a portion $S^{d-1} \times\left[-\tau_{0}, \tau_{0}\right]$ of the asymptotic Euclidean AdS boundary where to define the dual CFT, and therefore the existence of a CFT dual to our bulk construction. Intuitively, if $\tau_{0}<0$, the brane in Fig. 4 would overlap itself, leaving no portion of the asymptotic boundary in the geometry.

When both the conditions are satisfied, the properties of the resulting braneworld cosmology are encoded in the dual BCFT state, and can be extracted in principle by measuring appropriate observables. For example, at early times the Ryu-Takayanagi surface [58,59] associated with large regions of the CFT ends on the ETW brane, and therefore the entanglement entropy of such regions has a time dependence that probes the brane evolution [10]. This is analogous to what happens in doubly holographic setups

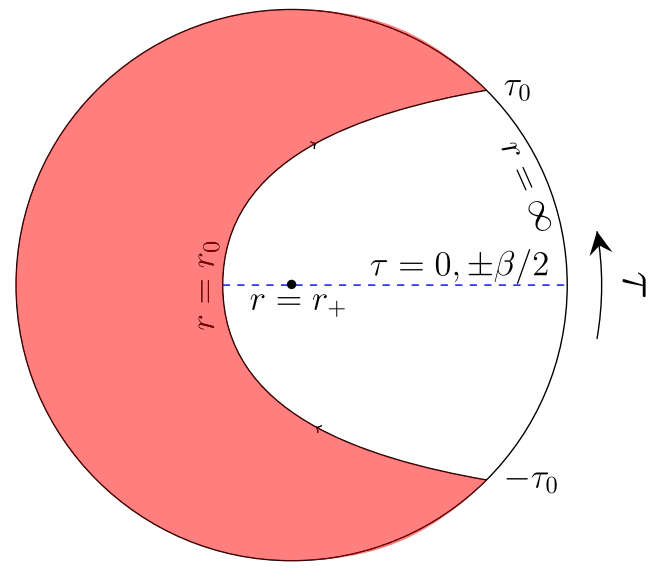

FIG. 4. Trajectory of the brane in the Euclidean wormhole. The angular coordinate is the Euclidean time $\tau$ and the radial coordinate is the radius $r$. The central point represents the outer horizon $r=r_{+}$and the circumference is the asymptotic AdS boundary. Each point in the diagram is a $(d-1)$-dimensional sphere. The ETW brane contracts from the boundary to a minimum radius $r_{0}$ at $\tau= \pm \beta / 2$, and then expands back to the boundary. The red region is outside the ETW brane and therefore is not part of the geometry.

involving an evaporating black hole on the brane [60], and suggests a connection between the braneworld models studied here and the physics of entanglement islands $[15-17,61,62]$.

The models reviewed in this section provide a framework to describe cosmology in AdS/CFT correspondence and therefore study quantum gravity in a cosmological universe. However, it is worth noting that in the simplest possible realization of this bottom-up proposal, involving an AdSSchwarzschild black hole and no matter in the bulk, conditions 1 and 2 cannot be simultaneously satisfied: when the brane is far enough to allow gravity localization, it also overlaps itself, violating condition 1 [10]. Including a bulk gauge field, and therefore considering an AdS-RN black hole, the two conditions can be satisfied at the same time, provided that the black hole is large and near extremal $\left(r_{-} \lesssim r_{+}\right)$. The corresponding saddle-point solution is also the dominant one in the thermodynamical ensemble [11]. An alternative possible solution to the "overlap problem" was recently proposed in [15], where the bulk theory involves additional fields associated with the dual CFT, which is constructed by coupling two three-dimensional CFTs (which are joined in the bulk IR by a connected brane) using a fourdimensional CFT with many less degrees of freedom $\left(c_{4 D}<<c_{3 D}\right.$, with $c$ central charge).

An explicit realization of a model similar to the one described in this section was provided in [22] in the context of the $\mathrm{AdS}_{3} / \mathrm{CFT}_{2}$ duality. A first attempt to build a topbottom model of the present braneworld construction appeared recently in [15]. The CFT is taken to be $\mathcal{N}=4$ SYM theory on a three-dimensional manifold times an interval, coupled to two superconformal three- 


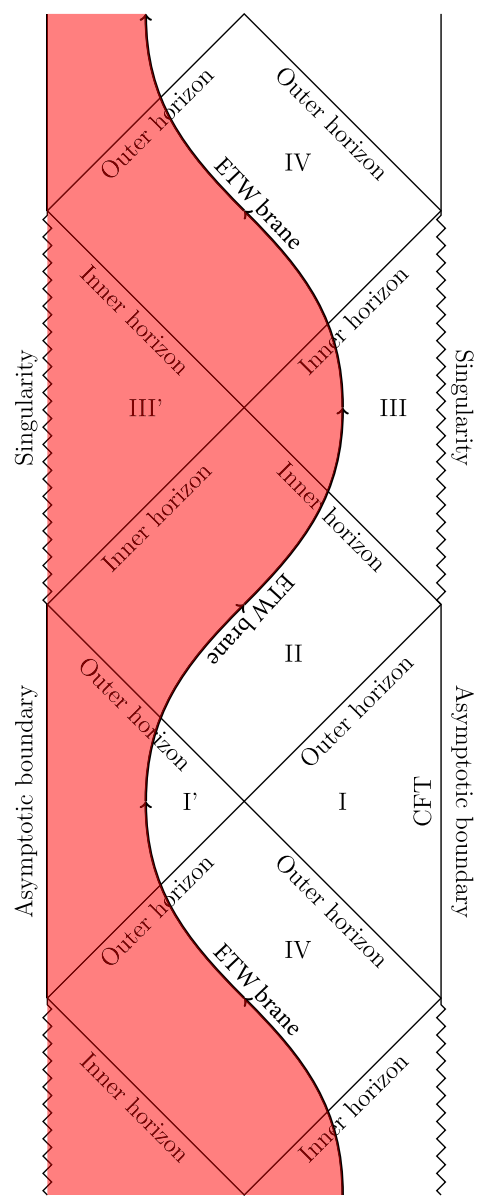

FIG. 5. Trajectory of the brane in Lorentzian signature. The brane emerges from the past horizon in the left asymptotic region and collapses into the future horizon. The red region, including the left asymptotic boundary, is cut off by the ETW brane and is not part of the geometry. We glued here more patches of the AdSRN spacetime, extending the trajectory of the brane. However, the trajectory is reliable only between the intersection points of the brane with the (inner) Cauchy horizon.

dimensional CFTs with many more degrees of freedom, living at the boundaries of the interval. The bulk dual picture is described by a specific configuration involving brane/antibrane pairs in type IIB string theory.

In the present paper, we are interested in a specific region of the parameter space of the AdS-RN bottom-up braneworld cosmology model [11]. In particular, we are interested in studying configurations where the minimum brane radius $r_{0}$ (in Euclidean signature) is just outside the horizon of a large, near-extremal black hole $r_{0} \gtrsim r_{+} \gtrsim r_{-} \gg L_{\text {Ads }}$. This setup, which we analyze in detail in the next section, is clearly not relevant for cosmology, since local gravity localization can be achieved only when $r_{0} \gg r_{+}$. However, it is well known [25,26,31-34] that the physics of the near-horizon region of a near-extremal AdS-RN black hole is well captured by 2D JT gravity, whose semiclassical description is in turn dual to the low-energy limit of the SYK (or cSYK) model. Therefore, when the brane enters the near-horizon region of the higher-dimensional black hole, we will be able to obtain by dimensional reduction an effective two-dimensional description involving an "end-of-the-world particle" (a one-dimensional ETW brane) cutting off part of the hyperbolic disk (i.e., Euclidean $\mathrm{AdS}_{2}$ ). The dynamics in such geometry is governed by JT gravity, and the dual description of specific configurations is given by the cSYK boundary states described in Sec. III.

Although this construction does not provide an example of braneworld cosmology in AdS/CFT correspondence, the simplicity of our setup allows us to gain insight about the structure of the duality between black hole spacetimes involving an ETW brane and specific boundary states in a dual holographic quantum mechanical theory.

\section{BULK DUAL OF CSYK BOUNDARY STATES}

The geometry of the near-horizon region of a $(d+1)$ dimensional near-extremal AdS-RN black hole approaches $\mathrm{AdS}_{2} \times S^{d-1}[25,26,31-34]$. At the low-energy scales we are interested in in our analysis, spherical perturbations are dominant [31] and the dynamics is well captured by an effective two-dimensional theory obtained by dimensional reduction. As usual when performing dimensional reduction, a dilaton field $\phi(\tau, r)$ is introduced, which plays the role of the radius of the $(d-1)$-dimensional sphere $S^{d-1}$ on shell. Away from the black hole horizon $r=r_{+}$, there are deviations from the product geometry $\mathrm{AdS}_{2} \times S^{d-1}$, and the dimensionally reduced model describes nearly $\mathrm{AdS}_{2}$ gravity. At leading order (which is near to the black hole horizon) such deviations are encoded in the fluctuations of a regularized boundary, and the physics is well approximated by JT gravity $[25,26,31,63]$.

In this section, we report the results ${ }^{9}$ of the dimensional reduction of the bottom-up holographic braneworld cosmology model reviewed in Sec. IV. We will find that, under appropriate conditions, the resulting theory mimics the properties of the cSYK boundary states we described in Sec. III, providing evidence for the duality between such boundary states and JT gravity setups involving an ETW particle. ${ }^{10}$

\section{A. Dimensional reduction of braneworld cosmologies}

We focus our attention on a fixed-charge canonical ensemble with charge $\mathcal{Q}$ [11]. Before dimensionally reducing the corresponding braneworld cosmology action, it is useful to perform a change of coordinates:

\footnotetext{
${ }^{9}$ Detailed calculations and additional considerations are reported in Appendix C

${ }^{10}$ In the late development stages of this work Ref. [64] appeared, where the authors explore the semiclassical and quantum properties of JT gravity setups involving ETW branes in Lorentzian signature. The results obtained there are complementary to the ones achieved in the present paper.
} 


$$
r \rightarrow r_{e}+\frac{R_{2}^{2}}{z}
$$

where $r_{e}$ is the horizon radius of the extremal black hole with charge $\mathcal{Q}$ [11] and we conveniently introduced the quantity

$$
R_{2}=\frac{r_{e} L_{\mathrm{AdS}}}{\sqrt{d(d-1) r_{e}^{2}+(d-2)^{2} L_{\mathrm{AdS}}^{2}}}
$$

which will be the $\mathrm{AdS}_{2}$ radius of the dimensionally reduced theory. The near-horizon region is then identified by the condition $z \gg R_{2}^{2} / r_{e}$. For the near-extremal case of our interest $r_{+} \gtrsim r_{e}$, after a Weyl rescaling the metric of the near-horizon region reads

$d s^{2} \approx \frac{R_{2}^{2}}{z^{2}}\left[\left(1-4 \pi T^{2} z^{2}\right) d t^{2}+\frac{d z^{2}}{\left(1-4 \pi T^{2} z^{2}\right)}\right]+r_{e}^{2} d \Omega_{d-1}^{2}$,

where $T=1 / \beta$ is the Hawking temperature of the black hole and the horizon is at $z_{H}=1 /(2 \pi T)$. In the extremal limit $T \rightarrow 0$ the two-dimensional metric reduces to Poincaré AdS. ${ }^{11}$ The two-dimensional metric in the near- extremal case (40) is still $\mathrm{AdS}_{2}$, and can be mapped to Poincaré AdS by a change of coordinates [31].

As we have already mentioned, the asymptotic $\mathrm{AdS}_{2}$ boundary must be regularized to the near-horizon region to cut off any large deviation from the $\mathrm{AdS}_{2} \times S^{d-1}$ geometry. When the black hole is large, $r_{+} \gtrsim r_{e} \gg L_{\mathrm{AdS}}$, it is possible to place the regularized boundary in the near-horizon region $z \gg R_{2}^{2} / r_{e}$ and at the same time deep in the asymptotic $\mathrm{AdS}_{2}$ region $z \ll R_{2} \approx L_{\mathrm{AdS}}$ [33]. In this region, corrections above extremality are also small if the temperature satisfies $2 \pi T \lesssim 1 / R_{2}$. The region where the regularized boundary sits is then defined by the conditions

$$
2 \pi T R_{2}^{2} \lesssim R_{2} \approx L_{\mathrm{AdS}} \ll \frac{R_{2}^{2}}{z} \ll r_{e} .
$$

We can now perform the dimensional reduction of the holographic braneworld cosmology action ([11], and reported in Appendix C). Working at fixed charge, we can further integrate out the bulk gauge field [32] to obtain the following dimensionally reduced effective action describing the physics inside the regularized boundary (the expressions of $S_{0}$ and $S_{0}^{\mathrm{ETW}}$ are reported in Appendix C):

$$
\begin{aligned}
S_{\mathrm{tot}}= & S_{0}+S_{0}^{\mathrm{ETW}}-\frac{V_{d-1}}{16 \pi G} \int_{\mathcal{M}} d^{2} x \sqrt{g} \Phi_{1}\left[R+\frac{2}{R_{2}^{2}}\right]-\frac{V_{d-1}}{8 \pi G} \int_{\partial \mathcal{M}_{\infty}} d u \sqrt{\gamma_{u u}} \Phi_{1} K^{\infty} \\
& -\frac{V_{d-1}}{8 \pi G} \int_{\mathrm{ETW}} d v \sqrt{h_{v v}} \Phi_{1}\left[K^{\mathrm{ETW}}-\frac{d}{2} \Phi_{0}^{-\frac{d-2}{2(d-1)}} T_{\mathrm{ETW}}\right]+\cdots
\end{aligned}
$$

$S_{0}$ is a topological term, $S_{0}^{\text {etw }}$ is a term proportional to the proper length of the trajectory of the ETW particle, $V_{d-1}$ is the volume of the $(d-1)$-dimensional unit sphere, $G$ is the $(d+1)$-dimensional Newton constant, $\mathcal{M}$ is the twodimensional spacetime manifold, $g_{i j}$ is the two-dimensional Weyl-rescaled metric, $\Phi_{1}$ is a redefined dynamical dilaton, $R$ is the two-dimensional Ricci scalar. $\partial \mathcal{M}_{\infty}$ is the regularized boundary, $\gamma_{u и}$ the metric induced on it, and $K_{\infty}$ its extrinsic curvature. Finally, $h_{v v}$ is the metric induced on the ETW particle, $K^{\mathrm{ETW}}$ its extrinsic curvature, and $\Phi_{0}=r_{e}^{d-1}$. The last two terms of the first line are the JT gravity action, while the second line is the ETW particle action, and the dots account for higher-order corrections away from the near-horizon region.

From the action (42) it is clear that the bulk geometry is fixed on shell to be $\mathrm{AdS}_{2}$, with $\mathrm{AdS}$ radius $R_{2}$. Euclidean $\mathrm{AdS}_{2}$ possesses an asymptotic $\operatorname{Diff}\left(S^{1}\right)$ boundary time reparametrization symmetry. Being an emergent

\footnotetext{
${ }^{11}$ Note that in Euclidean signature Poincaré coordinates cover the whole hyperbolic disk [26].
}

asymptotic symmetry, it is spontaneously broken by $\mathrm{AdS}_{2}$, and only a $\mathrm{SL}(2, \mathbb{R})$ subgroup remains unbroken [25]. As we will see in the next section, all the dynamics is encoded in the fluctuations of the boundary $\partial \mathcal{M}_{\infty}$. The leading-order fluctuations are described by a Schwarzian action that explicitly breaks the $\operatorname{Diff}\left(S^{1}\right)$ symmetry down to $\operatorname{SL}(2, \mathbb{R})$.

\section{B. Boundary conditions and Schwarzian action}

The action (42) must be supported by a set of boundary conditions for the metric and the dilaton at the regularized boundary and at the location of the ETW particle. At the regularized boundary, we impose the Dirichlet-Dirichlet (DD) boundary conditions usually implemented in JT gravity (see [65] for an analysis of the possible boundary conditions in JT gravity):

$$
\gamma_{u u}=\frac{1}{\varepsilon^{2}},\left.\quad \Phi_{1}\right|_{\partial \mathcal{M}_{\infty}}=\frac{\phi_{b}}{\varepsilon,}
$$

where $\varepsilon$ is a small cutoff parameter determining the location of the regularized boundary (whose total proper length is 
set to be $L=\beta / \varepsilon$ ) and $\phi_{b}$ is a constant. On the other hand, according to the AdS/BCFT prescription $[7,8]$ and to the higher-dimensional braneworld cosmology model reviewed in Sec. IV, we must impose NeumannNeumann (NN) boundary conditions on the ETW particle. They are given by (a derivation of these boundary conditions is given in Appendix D)

$$
\begin{gathered}
K^{\mathrm{ETW}}-\frac{d}{2} r_{e}^{-\frac{d-2}{2}} T_{\mathrm{ETW}}=0 \\
n_{\mathrm{ETW}}^{i} \partial_{i} \Phi_{1}-\Phi_{1} K^{\mathrm{ETW}}-(d-1) r_{e}^{\frac{d}{2}} T_{\mathrm{ETW}}=0 .
\end{gathered}
$$

We can now obtain an effective action governing the dynamics of the gravitational theory by integrating out the dilaton field $\Phi_{1}$, evaluating the action on shell, and imposing the boundary conditions (43), (44), and (45). This fixes the bulk geometry to be $\mathrm{AdS}_{2}$, with the metric given by the two-dimensional part of Eq. (40).

The only dynamical term left in the effective action comes from the GHY term for the regularized boundary $\partial \mathcal{M}_{\infty}$. Parametrizing the boundary with the boundary proper time $u$, the location of the boundary in the fixed Euclidean $\mathrm{AdS}_{2}$ bulk is given by $(\tau(u), z(u))$, with $\tau$ bulk time coordinate. Up to third order in $\varepsilon$, the boundary conditions (43) impose

$z(u) \approx \varepsilon R_{2} \tau^{\prime}(u)+\varepsilon^{3} R_{2}^{3}\left[\frac{\left(\tau^{\prime \prime}(u)\right)^{2}}{2\left(\tau^{\prime}(u)\right)^{2}}-2 \pi^{2} T^{2}\left(\tau^{\prime}(u)\right)^{3}\right]$,

where a prime indicates a derivative with respect to the boundary proper time $u$. The shape of the regularized boundary is then completely determined by the time reparametrization mode $\tau(u)$, and the dynamics is encoded in its fluctuations, which are described at leading order in $1 / \varepsilon$ by a Schwarzian action:

$$
I_{\text {eff }} \approx-\frac{V_{d-1} R_{2} \phi_{b}}{8 \pi G} \int d u\{\tan (\pi T \tau(u)), u\} .
$$

In the absence of the ETW particle, the integral over $u$ runs from $-\beta / 2$ to $\beta / 2$ and $\tau(u)$ is a monotonic function satisfying $\tau(u+\beta)=\tau(u)+\beta$. The action (47) explicitly breaks the asymptotic $\operatorname{Diff}\left(S^{1}\right)$ symmetry down to $\mathrm{SL}(2, \mathbb{R})$. This pattern of symmetry breaking is completely analogous to the one found in the SYK model, and makes the duality between low-energy limit of the SYK model and JT gravity manifest. In particular, the gravitational Schwarzian sector can be described either by the Majorana SYK model, or by a fixed-charge subsector of the cSYK model. The picture emerging from the analysis so far is that one of a rigid $\mathrm{AdS}_{2}$ bulk cutoff by a regularized boundary whose shape is determined by the function $\tau(u)$ (see Fig. 6). The Schwarzian action (47) takes different values on "chunks" of $\mathrm{AdS}_{2}$ with different shapes, ${ }^{12}$ which

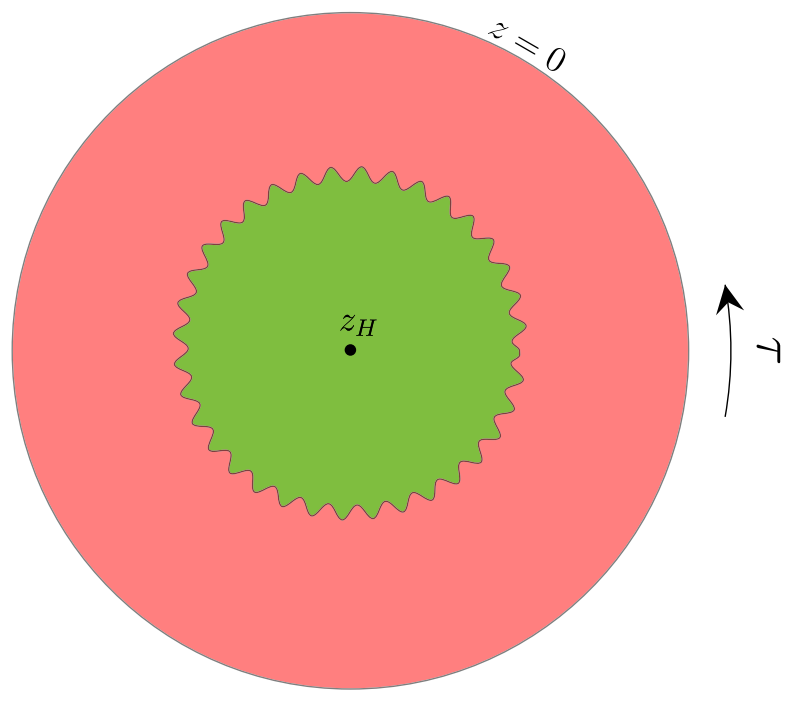

FIG. 6. Time reparametrization and $\mathrm{AdS}_{2}$ chunks. Different time reparametrizations $\tau(u)$ correspond to different shapes of the regularized boundary in the rigid $\mathrm{AdS}_{2}$ bulk. The spacetime region described by our effective action is the one enclosed by the regularized boundary, and shaded in green. Chunks of $\mathrm{AdS}_{2}$ of different shapes are weighted differently by the Schwarzian action in the path integral.

are then weighted differently in the gravitational path integral. The saddle-point solution is given by $\tau(u)=u$, which represents a circular boundary sitting at $z=\varepsilon R_{2} \equiv z_{b}$.

\section{ETW particle trajectory}

So far we have focused on the dynamics of the regularized boundary. Imposing the boundary conditions (44) and (45) the action for the ETW particle clearly vanishes on shell. Therefore, in the resulting effective description the ETW particle follows a trajectory (fixed by the boundary conditions) in the rigid $\mathrm{AdS}_{2}$ bulk, and its evolution is independent of the regularized boundary fluctuations, i.e., of the dynamics. Parametrizing the ETW particle worldline by the bulk time $\tau$ [i.e., picking $v=\tau$ in the action (42)], the equation of motion for the brane particle is given by

$$
\begin{aligned}
z^{\prime}(\tau)= & \pm \frac{1-4 \pi^{2} T^{2} z^{2}}{T_{\mathrm{ETW}}\left(\frac{d R_{2}}{2}+\frac{r_{e}}{R_{2}} z\right)} \\
& \times \sqrt{1-4 \pi^{2} T^{2} z^{2}-\left(\frac{d R_{2}}{2}+\frac{r_{e}}{R_{2}} z\right)^{2} T_{\mathrm{ETW}}^{2}}
\end{aligned}
$$

where the $+(-)$ sign corresponds to the contraction (expansion) phase of the ETW particle. As described in Sec. IV, we are interested in time-reflection symmetric

\footnotetext{
${ }^{12} \mathrm{Up}$ to an $\mathrm{SL}(2, \mathbb{R})$ transformation connecting physically equivalent configurations.
} 
states. Therefore, the brane reaches its minimum radius (i.e., maximum value of $z$ ) $z=z_{\max }$ for $\tau= \pm \beta / 2 . z=$ $z_{\max }$ is the inversion point $z^{\prime}( \pm \beta / 2)=0$, and is determined by imposing the argument of the square root in Eq. (48) to vanish. If we could trust this trajectory all the way to the asymptotic $\mathrm{AdS}_{2}$ boundary, it would describe a particle hitting the asymptotic boundary at times $\tau= \pm \bar{\tau}$, and reaching its minimum radius at the inversion point $z_{\max }$. However, we remark that the trajectory described by Eq. (48) is reliable only inside the regularized boundary, where the physics is well described by the dimensionally reduced action (42).

Since our effective two-dimensional description is cut off at the regularized boundary, the ETW particle is present in our model only if it crosses the boundary and enters the near-horizon region. At leading order in $\varepsilon$ this implies

$$
z_{\max } \geq \varepsilon R_{2} \tau^{\prime}(u)=z_{b} \tau^{\prime}(u) .
$$

For $\tau(u)=u$, condition (49) imposes a bound on the tension $T_{\mathrm{ETW}}$ :

$$
0<T_{\mathrm{ETW}} \leq T_{\mathrm{ETW}}^{\mathrm{max}}=\frac{\sqrt{1-4 \pi^{2} T^{2} z_{b}^{2}}}{\frac{d R_{2}}{2}+\frac{r_{e}}{R_{2}} z_{b}} .
$$

Using the conditions (41) determining the region where the regularized boundary sits, we immediately find that Eq. (50) implies $0<T_{\mathrm{ETW}} \ll 1 / L_{\mathrm{AdS}}$, which means that the tension of the higher-dimensional ETW brane must be far from its critical value. This result was to be expected because in the higher-dimensional picture we want the ETW brane to enter the near-horizon region.

The near-horizon condition also guarantees that in the portion of trajectory we are interested in (i.e., when the ETW particle is inside the regularized boundary) $z(\tau) \gg$ $R_{2}^{2} / r_{e}$ holds. In this limit, the equation of motion (48) can be solved analytically, obtaining

$$
\begin{aligned}
z(\tau) \approx & \frac{R_{2}}{\sqrt{4 \pi^{2} T^{2} R_{2}^{2}+r_{e}^{2} T_{\mathrm{ETW}}}} \\
& \times \sqrt{1-\frac{r_{e}^{2} T_{\mathrm{ETW}}}{4 \pi^{2} T^{2} R_{2}^{2}} \tan ^{2}\left[\frac{2 \pi}{\beta}\left(\tau \mp \frac{\beta}{2}\right)\right]},
\end{aligned}
$$

and now $z_{\max } \approx R_{2} / \sqrt{4 \pi^{2} T^{2} R_{2}^{2}+r_{e}^{2} T_{\mathrm{ETW}}^{2}}$, while the maximum brane tension is $T_{\mathrm{ETW}}^{\mathrm{max}} \approx R_{2} \sqrt{1-4 \pi^{2} T^{2} z_{b}^{2}} /\left(z_{b} r_{e}\right)$.

\section{Tangent trajectory and symmetry breaking}

Let us initially focus on the Schwarzian saddle point $\tau(u)=u$. In this case the regularized boundary is circular and sits at $z=z_{b}$. When the condition (49) is satisfied, the brane will in general intersect the boundary in two points, at times $\tau=u= \pm u_{0}$, with $u_{0}<\beta / 2$. The AdS/BCFT prescription suggests that the quantity $u_{0}$ represents the preparation time of the state dual to the spacetime geometry. Although this solution is completely meaningful on the gravity side, it is not clear at the moment what the dual cSYK state would look like. Indeed, the preparation time of the boundary states introduced in Sec. III is by construction $u_{0}=\beta / 2$.

One possibility is that the geometry with two intersection points is dual to some different kind of cSYK boundary state, one with a different structure at the microscopic scale. This is perfectly reasonable since the brane intersection with the regularized boundary is by definition at the cutoff scale in the dimensionally reduced theory.

It is also conceivable that only holographic boundary states with preparation time $u_{0}=\beta / 2$ can be built in the cSYK model, and that the two-intersection solutions are only an indication that the bulk theory is richer than the boundary one. This would not come as a surprise for two reasons. First, the analogy with the $\mathrm{AdS}_{3} / \mathrm{CFT}_{2}$ case, where only holographic boundary states with preparation time $u_{0}=\beta / 4$ (and therefore the brane anchored at antipodal points) can be constructed using conformal boundary conditions [22]. Second, the fact that the bulk theory described in the present section comes from the dimensional reduction of a higher-dimensional theory, and is therefore expected to be richer than the $(0+1)$-dimensional boundary theory. This is made explicit by the presence of an additional bulk parameter, the tension $T_{\text {ETW }}$, which has no known counterpart in our cSYK description. In higher-dimensional braneworld cosmologies, the bulk tension parameter has a counterpart in the boundary dual theory, with a precise physical meaning: it accounts for additional CFT degrees of freedom living on the $(d-1)$-dimensional boundary of the $d$-dimensional manifold where the BCFT is defined $[10,15]$. But the dual theory considered in the present paper is $(0+1)$-dimensional quantum mechanics, and therefore there can be no additional CFT boundary degrees of freedom. The tension is then just a bulk free parameter inherited from the higherdimensional description.

To make contact with the boundary states defined in Sec. III, we therefore impose $u_{0}=\beta / 2$, which implies that the two intersection points reduce to a single one at bulk coordinates $\tau= \pm u_{0}= \pm \beta / 2$ and $z=z_{\max }=z_{b}$ (see Fig. 7). This condition can be met for a brane trajectory sufficiently close to the horizon provided we choose the cutoff surface $z_{b}$ such that $T_{\mathrm{ETW}}=T_{\mathrm{ETW}}^{\mathrm{max}}\left(z_{b}\right)$. In other words, the trajectory of the particle is tangent to the regularized boundary. One then has a special point on the regularized boundary where Neumann boundary conditions for all the bulk fields are imposed, which is reminiscent of the cSYK thermal circle with a special point. If we took into account the presence of bulk fermions in our description, appropriate boundary conditions for the 


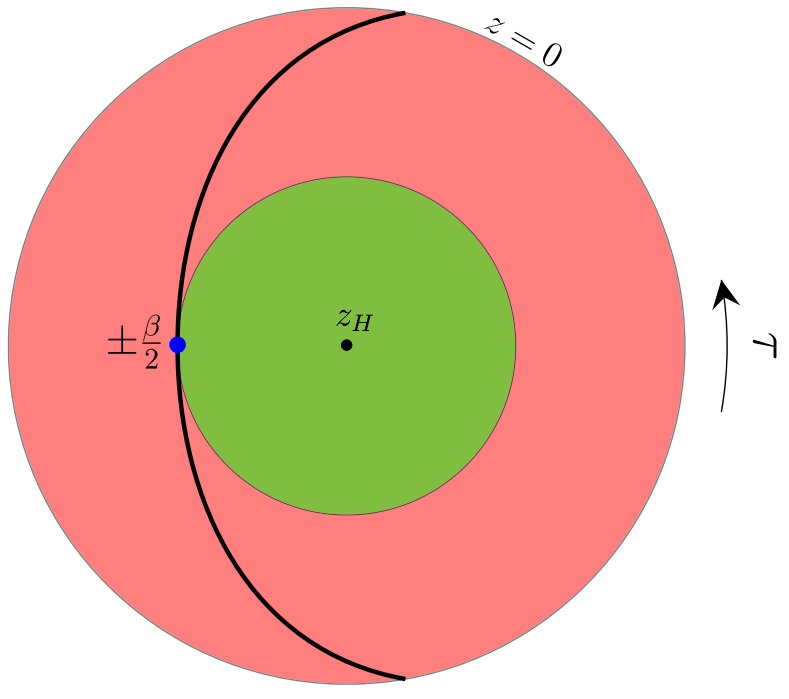

FIG. 7. Tangent ETW particle at saddle point. At the Schwarzian saddle point $\tau=u$ the regularized boundary is a circle sitting at $z=z_{b}$. When the ETW particle trajectory is tangent to the regularized boundary, a single special point at $\tau= \pm \beta / 2$ is identified, where Neumann boundary conditions for all the bulk fields must be imposed. This configuration is the one relevant to the description of the cSYK boundary states analyzed in Sec. III.

fermions should also be specified at the special point. Let us now consider dynamical fluctuations of the regularized boundary, governed by the effective action (47). From a bulk point of view, we could consider arbitrary boundary reparametrizations $u \rightarrow \tau(u)$, i.e., arbitrary shapes for the regularized boundary in the rigid $\mathrm{AdS}_{2}$ bulk (see Fig. 6). The single intersection point at $\tau=u= \pm \beta / 2$ can then be mapped to a different single intersection point at an arbitrary $\tau(\beta / 2)=\tau(-\beta / 2)+\beta$, and it would not reside on the time-reflection symmetric line $\tau= \pm \beta / 2$ anymore. Or there could be more than one intersection point, or no intersection points at all. We know of no principle restricting such configurations from the bulk point of view, and this is again a sign of the additional freedom present in the higherdimensional bulk theory relative to the lower-dimensional boundary dual. But since our goal is to build a geometry which can be described by the cSYK boundary states defined in Sec. III, a necessary condition for our construction to work is that both the time-reflection symmetry of the ETW particle trajectory in the bulk and the tangent condition must be preserved under reparametrizations of the boundary. In other words, the intersection of the ETW particle trajectory and the boundary must remain a single point on the time-reflection symmetric line $\tau= \pm \beta / 2$.

The time-reflection symmetry condition immediately implies that the intersection point $u= \pm \beta / 2$ is mapped by an admissible boundary reparametrization to $\tau( \pm \beta / 2)= \pm \beta / 2$. Since the trajectory of the ETW particle in the bulk is completely independent of the boundary fluctuations, $\quad z_{\mathrm{ETW}}(\tau= \pm \beta / 2)=z_{\max }=z_{b} \quad$ still holds after the boundary reparametrization. By means of Eq. (46), at leading order in $\varepsilon$ the tangent condition implies $z(u= \pm \beta / 2)=z_{b} \tau^{\prime}( \pm \beta / 2)=z_{b}$, and therefore $\tau^{\prime}( \pm \beta / 2)=1$. Note that since the Schwarzian fluctuations are $1 / N$ suppressed (which is of Planckian size), the preservation of the tangent condition under time reparametrizations also implies that no additional intersection points appear in perturbation theory: the special point at $\tau= \pm \beta / 2$ remains the only intersection point between the ETW particle trajectory and the regularized boundary. After the identification $u_{0}=\beta / 2$, we obtain two boundary conditions for the boundary reparametrization mode:

$$
\tau\left( \pm u_{0}\right)= \pm u_{0}, \quad \tau^{\prime}\left( \pm u_{0}\right)=1
$$

which are completely analogous to the ones obtained for the cSYK boundary states [see Eq. (31)]. Therefore we obtained the exact same pattern of symmetry breaking: the effect of the boundary conditions (52) is to further break the residual $\operatorname{SL}(2, \mathbb{R})$ symmetry, with only one generator left unbroken. This result provides clear evidence of the duality between the cSYK boundary states constructed in Sec. III and the dimensionally reduced braneworld cosmology model described in the present section.

As we have already explained at the end of Sec. III C, the boundary states described in Sec. III are not the only ones to exhibit the properties described in the present analysis: starting with a generic superposition of Fock states (instead of a single Fock state) and evolving it for an amount $\tau_{0}$ of Euclidean time would lead to analogous results. ${ }^{13}$ Note, however, that a complete dual description of the low-energy limit of the cSYK model must necessarily include $N$ bulk fermions, related to sources of the cSYK fermions by the HKLL construction [50]. A set of boundary conditions for such bulk fields must be specified at the intersection point between the regularized boundary and the ETW particle. A given set of boundary conditions will then uniquely identify a specific cSYK boundary state from a bulk perspective. From this point of view, the construction described in the present section, where we ignored the presence of the bulk fermions, can be regarded as the common dual geometric background associated with the whole class of cSYK boundary states that break the $\operatorname{SL}(2, \mathbb{R})$ symmetry in the specific way we analyzed. It is then a conceptually straightforward step to specify a set of boundary conditions for the $N$ bulk fields at the intersection point, and therefore restrict to a single cSYK boundary state.

\footnotetext{
${ }^{13}$ Note that this is not true in general for a highly excited state. As an example, consider an energy eigenstate. The eigenstate thermalization hypothesis guarantees that correlators of generic operators would look thermal in such state. However, it is enough to compute quantum energy fluctuations to tell apart an energy eigenstate (for which they would exactly vanish) from the thermal state. This is different from the expectations for our boundary states and could be a signal that energy eigenstates do not have a smooth interior.
} 
We emphasize that we only used the intuition provided by the cSYK boundary states as a guideline to understand the symmetry properties that a hypothetical bulk dual should satisfy. The fact that requiring such properties to hold in our dimensionally reduced braneworld cosmology model leads to the same symmetry-breaking pattern that we observed on the cSYK side is nontrivial, and should be regarded as a key result of the present paper.

\section{DISCUSSION}

In this paper we built an explicit example of the duality between asymptotically AdS spacetimes with an end-ofthe-world brane and boundary states of a dual CFT. In particular, we considered specific boundary states in the cSYK model and showed that, under appropriate conditions, the dimensional reduction of the holographic braneworld cosmology model proposed in [11] is dual to such boundary states. Although the range of bulk parameters where the duality holds does not allow gravity localization on the ETW brane (and therefore the model is not suitable to properly describe a braneworld cosmology), our result confirms the possibility to describe similar spacetimes using BCFTs and sheds light on how to explicitly realize similar constructions. It also has the merit to provide a novel, simple framework to explore the properties of the AdS/BCFT correspondence.

A direction calling for further development is to understand which observables in the cSYK model can probe the bulk trajectory of the ETW particle. In a Lorentzian picture corresponding to our Euclidean model, the ETW particle worldline would sit in the left asymptotic region, cutting off the left asymptotic boundary, analogously to what happens in the higher-dimensional model depicted in Fig. 5 (see [64] for a recent analysis). The duality between the cSYK boundary states and such geometries implies that the trajectory of the ETW particle in the left asymptotic region can be probed by measuring expectation values of appropriate observables in the boundary state of the cSYK model living on the right asymptotic boundary, which is dual to the whole spacetime. In particular, the expectation values should acquire some time dependence, which would necessarily be related to the evolution of the ETW particle in the bulk. Natural candidates to probe such behind-thehorizon physics are the entanglement entropy and the holographic complexity [10]. Our construction allows us to further extend this list of possible observables by working within a well-understood, controlled framework.

We also remark one more time that in the present paper we explored only the Schwarzian sector of the dimensionally reduced theory, and in particular we did not explicitly take into account bulk fermions, which are necessarily present in a bulk dual of the low-energy sector of the cSYK model. Therefore, the geometries we obtained can be equivalently described at this level by either the cSYK boundary states in a specific charge subsector constructed in Sec. III (or analogous states built by starting with a generic superposition of Fock states instead of a single Fock state), or the Majorana SYK boundary states studied by Kourkoulou and Maldacena [21]. Additionally, our purely Schwarzian bulk construction is incapable of distinguishing between two different boundary states in the same cSYK charge subsector (or any two boundary states in the Majorana SYK model). However, when the presence of bulk fermions (which are related to sources of the cSYK fermions by the HKLL construction [50]) is taken into account, the cSYK boundary states are uniquely identified by the specific set of boundary conditions imposed on the bulk fields at the intersection point between the ETW particle and the regularized boundary. Our bulk construction is then dual to a whole class of boundary states, which are distinguished by the boundary conditions imposed on bulk fermions propagating on such geometric background. One advantage of considering cSYK boundary states instead of Majorana SYK ones is that it allows an immediate generalization of our setup to more complicated configurations. For example, it would be possible to study the higherdimensional holographic braneworld cosmologies in the gravitational grand-canonical ensemble, allowing small charge fluctuations. The corresponding cSYK effective action would then also include a term governing the dynamics of the phase field $\lambda(\tau)$ and encoding small charge-density fluctuations [31]. Alternatively, we could include in our description $N$ bulk fermions charged under the $U(1)$ bulk gauge symmetry. These would be naturally described within the cSYK model [44,50] and would determine the specific cSYK boundary state dual to the spacetime geometry, as we have already pointed out.

Another interesting open question is whether or not it is possible to build a dual description of bulk setups where the brane intersects the regularized boundary in two distinct points. If the answer is yes, the corresponding boundary states should look quite different from the ones built in Sec. III, whose preparation time is by construction equal to half the Euclidean periodicity. It is also possible that such bulk setups are just a manifestation of the additional bulk degrees of freedom inherited from the higher-dimensional theory. If this is the case, they might not have a dual representation in the cSYK model.

Finally, a fascinating open question is how the holographic braneworld cosmology models originally introduced in $[10,11]$ relate to the physics of entanglement islands. The dimensionally reduced model presented in this paper could be a useful building block to explore the answer to this question in a simplified setup analogous to the ones where entanglement islands were originally introduced.

\section{ACKNOWLEDGMENTS}

We would like to thank J. Bringewatt, F. Ferretti, R. A. Silva, and J. L. Villaescusa Nadal for useful discussions. 
This work was supported in part by the U.S. Department of Energy, Office of Science, Office of High Energy Physics QuantISED Award No. DE-SC0019380, by the U.S. Department of Energy, Office of Science, Office of Advanced Scientific Computing Research, Accelerated Research for Quantum Computing program, and by the Simons Foundation via the It From Qubit collaboration.

\section{APPENDIX A: cSYK MODEL AND SYMMETRY BREAKING}

As we have pointed out in Sec. II, in general the saddlepoint solution (7) for $G$ is not invariant under the transformation (5), meaning that the time reparametrization symmetry $\operatorname{Diff}\left(S^{1}\right)$ and the local $U(1)$ symmetry are spontaneously broken. However, there is a subgroup of time reparametrizations under which the thermal solution is invariant provided that we also perform an appropriate phase shift [45]. Let us start by considering the zerotemperature solution (6) when the asymmetry parameter vanishes $\mathcal{E}=0$ (i.e., for the $\mathcal{Q}=0$ charge subsector). We can perform a time reparametrization

$$
\tau \rightarrow F(\tau)=\tan \left[\frac{\pi f(\tau)}{\beta}\right]
$$

without performing any local phase shift. Then, from Eq. (5) we obtain

$$
G_{f}\left(\tau_{1}, \tau_{2}\right) \propto\left(\frac{F^{\prime}\left(\tau_{1}\right) F^{\prime}\left(\tau_{2}\right)}{\left[F\left(\tau_{1}\right)-F\left(\tau_{2}\right)\right]^{2}}\right)^{\Delta} .
$$

For $f(\tau)=\tau$, this is the saddle-point thermal solution (7) at $\mathcal{E}=0$ [and it is correctly antiperiodic: $G_{\beta}(-\beta / 2)=$ $G_{\beta}(\beta / 2)$ ], while for generic $f(\tau)$ we get a generic reparametrization of the thermal solution. But note that Eq. (A2) is invariant under any reparametrization of the form

$$
\tilde{F}=\frac{a F+b}{c F+d}
$$

with $a d-b c=1$, i.e., the thermal solution is invariant under global $\operatorname{SL}(2, \mathbb{R})$ transformations. ${ }^{14}$ Note that the Möbius transformation (A3) does not distinguish elements in the center of $\operatorname{SL}(2, \mathbb{R})$. In other words, it does not distinguish the transformation with $a=d=1, b=c=0$ (i.e., the identity transformation $\hat{\mathbb{1}}$ ) from the one with $a=d=-1, b=c=0$ (i.e., the transformation $-\hat{\mathbb{1}}$ ). Therefore, looking only at the bosonic sector we are considering, the residual symmetry group is $\operatorname{PSL}(2, \mathbb{R})=\operatorname{SL}(2, \mathbb{R}) /\{ \pm \hat{\mathbb{1}}\}$. However, since in general we are interested in a bulk theory with fermions and

\footnotetext{
${ }^{14}$ It is a global $\operatorname{SL}(2, \mathbb{R})$ group because the transformation (A3) does not depend on $\tau$.
}

fermions are sensitive to the cover of $\operatorname{PSL}(2, \mathbb{R})$, we will consider $\operatorname{SL}(2, \mathbb{R})$ to be the residual symmetry group [48]. Therefore, the $\operatorname{Diff}\left(S^{1}\right)$ symmetry is spontaneously broken down to $\operatorname{SL}(2, \mathbb{R})$, and the corresponding pseudo-NambuGoldstone boson $f(\tau)$, whose dynamics is governed by the Schwarzian action, belongs to the left quotient of $\operatorname{Diff}\left(S^{1}\right)$ by $\operatorname{SL}(2, \mathbb{R})$. Note that the $\operatorname{SL}(2, \mathbb{R})$ symmetry is preserved by both the spontaneous and the explicit symmetry breaking, and must be regarded as a gauge symmetry as we explained in Sec. II. Indeed, the effective action (9) is invariant under the $\mathrm{SL}(2, \mathbb{R})$ transformation (A3). If we did not exclude the redundant $\operatorname{SL}(2, \mathbb{R})$-equivalent configurations when performing the path integral, we would have an infinite degeneracy leading to a diverging Euclidean path integral, as it always happens when we do not deal with gauge symmetries properly.

We also remark that any local phase shift does not leave either the thermal two-point or the effective action invariant: the emergent local $U(1)$ symmetry is completely broken, both spontaneously and explicitly, leading to a second pseudo-Nambu-Goldstone boson $\lambda(\tau)$, whose effective action governs charge fluctuations in the grandcanonical ensemble and is given by [44]

$$
I_{\mathrm{eff}}[\lambda, f]=\frac{N K}{2} \int_{0}^{\beta} d \tau\left[\lambda^{\prime}(\tau)+i \frac{2 \pi \mathcal{E}}{\beta} f^{\prime}(\tau)\right]^{2},
$$

where $K$ is the charge compressibility at zero temperature [44]. On the other hand, a global phase shift is an exact symmetry of the theory.

Let us now generalize our analysis to the case $\mathcal{E} \neq 0$. Now, due to the spectral asymmetry, we cannot obtain the thermal solution (7) from Eq. (6) by simply performing the time reparametrization (A1). Indeed, at the saddle point $f(\tau)=\tau$ we would not obtain an antiperiodic result with period $\beta$, as we would expect since $\tau$ and $\tau+\beta$ are identified. To restore the periodicity, we must also perform a local phase shift as in Eq. (5) with

$$
i \lambda(\tau)=-\frac{2 \pi \mathcal{E}}{\beta} \tau, \quad-\beta<\tau<\beta,
$$

which leads to the thermal two-point function (7). We see immediately that for $\mathcal{E} \neq 0$ such saddle-point solution, which transforms as in Eq. (5), is not invariant if we only perform a $\mathrm{SL}(2, \mathbb{R})$ transformation as defined by Eq. (A3). Indeed, the time reparametrization affects also the local phase shift (A5). However, the thermal two-point function is still invariant under the combined action of a $\operatorname{SL}(2, \mathbb{R})$ transformation and a local phase shift given by [45]

$$
i \bar{\lambda}(\tau)=\frac{2 \pi \mathcal{E}}{\beta}(f(\tau)-\tau) .
$$

Note that the total effective action [given by the sum of the Schwarzian action (9) and the phase field action (A4)] is 
invariant under the combined $\operatorname{SL}(2, \mathbb{R}) \otimes U(1)$ transformation. This is indeed equivalent to performing the change of variables

$$
\lambda(\tau) \rightarrow \lambda(\tau)+i \frac{2 \pi \mathcal{E}}{\beta}(\tau-f(\tau))
$$

which decouples the $U(1)$ mode from the time reparametrization mode, and then perform a $\operatorname{SL}(2, \mathbb{R})$ time reparametrization, which leaves invariant the actions for both modes. Substituting the change of variable (A7) into the transformation law (5) of the two-point function, we immediately see how a time reparametrization of the conformal thermal solution implies also a (imaginary) phase shift:

$$
\begin{aligned}
G_{f, \lambda}^{\beta}\left(\tau_{1}, \tau_{2}\right)= & {\left[f^{\prime}\left(\tau_{1}\right)\right]^{\Delta}\left[f^{\prime}\left(\tau_{2}\right)\right]^{\Delta} \mathrm{e}^{i\left[\lambda\left(\tau_{1}\right)-\lambda\left(\tau_{2}\right)\right]} } \\
& \times \mathrm{e}^{\frac{2 \pi \xi}{\beta}\left[f\left(\tau_{1}\right)-f\left(\tau_{2}\right)-\left(\tau_{1}-\tau_{2}\right)\right]} G_{\beta}\left(f\left(\tau_{1}\right), f\left(\tau_{2}\right)\right) .
\end{aligned}
$$

The transformation law (A8) leaves the (anti)periodicity of the thermal two-point function unchanged. Moreover, it ensures that a time reparametrization satisfying (A3) leaves the thermal solution invariant, making the $\operatorname{SL}(2, \mathbb{R})$ symmetry explicit again without the need of a local phase shift. Now all the considerations made in the $\mathcal{E}=0$ case apply here too, and the system displays the same pattern of symmetry breaking. In particular, the time reparametrization symmetry is both spontaneously and explicitly broken down to $\operatorname{SL}(2, \mathbb{R})$, which is a residual and nonphysical "gauge" symmetry, while the local $U(1)$ symmetry is completely broken both spontaneously and explicitly to global $U(1)$. Note indeed that a global phase shift still leaves the thermal two-point function invariant, i.e., there is still correctly an unbroken $U(1)$ global symmetry. Such global phase shift clearly also leaves the total effective action invariant: it represents an exact physical symmetry of the theory.

Finally, we would like to remark one more time that the boundary conditions (31) do not imply the exclusion of any physical configuration from the path integral. Indeed, we have seen that their effect is to further break two out of the three $\operatorname{SL}(2, \mathbb{R})$ generators. This implies that in general a $\mathrm{SL}(2, \mathbb{R})$ transformation (A3) maps modes that satisfy the boundary conditions (31) to modes that do not, and vice versa. In particular, for any given reparametrization mode $\bar{f}(\tau)$ which does not satisfy the boundary conditions, it is always possible to perform an appropriate $\operatorname{SL}(2, \mathbb{R})$ transformation to a mode $\tilde{f}(\tau)$ which satisfies (31). Since time reparametrization modes connected by the $\mathrm{SL}(2, \mathbb{R})$ transformation (A3) are accounted for only once in the path integral, the effect of the boundary conditions (31) is to select a one-parameter family of admissible reparametrization modes for each class of $\mathrm{SL}(2, \mathbb{R})$-equivalent modes.

\section{APPENDIX B: BOUNDARY STATES DYNAMICS}

\section{Probability distribution of coefficients}

In Sec. III we made use of the properties (outlined in Sec. III A) of the probability distribution of the coefficients $c_{k}^{i}$ defined by $\left|S_{i}\right\rangle=\sum_{k=1}^{D_{\mathcal{Q}}} c_{k}^{i}\left|E_{k}\right\rangle$. We report here physical motivations and numerical results supporting the features claimed in Sec. III A.

An intuitive way to understand this is by focusing on the contribution of each Fock state to the superposition $\left|E_{k}\right\rangle=$ $\sum_{i=1}^{D_{\mathcal{Q}}} c_{i}^{k}\left|S_{i}\right\rangle$ [with $c_{i}^{k}=\left(c_{k}^{i}\right)^{*}$ ] for multiple realizations of the random Hamiltonian. The emergent $U(N)$ symmetry at large $N$, arising from the properties of the random couplings and mentioned in Sec. III A, implies that the roles of the different Fock states are "shuffled" from one realization to another. Therefore, we expect the coefficient to have, on average, the same magnitude and a uniformly distributed phase. The value of the average magnitude is fixed by the normalization condition to be $1 / D_{\mathcal{Q}}$ (see Figs. 8 and 9 ).

The simplest model which captures these intuitions takes the coefficients $c_{k}^{i}$ as elements of a normalized Haar random vector in a $D_{\mathcal{Q}}$-dimensional complex vector space. Given such a random state $|\psi\rangle$, we have the standard identity

$$
\int d \psi|\psi\rangle\langle\psi|\otimes| \psi\rangle\langle\psi|=\frac{\mathbb{1}_{2}+F_{2}}{D_{\mathcal{Q}}\left(D_{\mathcal{Q}}+1\right)},
$$

where $\mathbb{1}_{2}$ is the identity on two copies and $F_{2}$ is the swap operator. Based on this formula, a random vector model of the $c_{k}^{i}$ predicts that the variance of $\left|c_{k}^{i}\right|^{2}$ is $D_{\mathcal{Q}}^{-2}+\mathcal{O}\left(D_{\mathcal{Q}}^{-3}\right)$ and that the covariance of $\left|c_{k}^{i}\right|^{2}$ and $\left|c_{l}^{i}\right|^{2}$ is $-D_{\mathcal{Q}}^{-3}+\mathcal{O}\left(D_{\mathcal{Q}}^{-4}\right)$.

Our numerical results, reported in Fig. 10, show that the variance of the coefficients is indeed close to $D_{\mathcal{Q}}^{-2}$. Moreover, the numerical data reported in Fig. 11 show a covariance close to $-D_{\mathcal{Q}}^{-3}$. Finally, since all the reasoning above can be applied indistinctly to any energy eigenstate in the charge subsector, the correlation between the energy eigenvalues and the coefficients is also strongly suppressed (see Fig. 12).

Our numerical results have been obtained using exact diagonalization for $N=8$, and averaging over 5000 realizations of the Hamiltonian. Increasing the number of realizations the points converge to the average values indicated by the horizontal lines, which are therefore very close to the true probability distribution's moments considered. We report here the results for the $\mathcal{Q}=0,-1,2$ charge subsectors. 


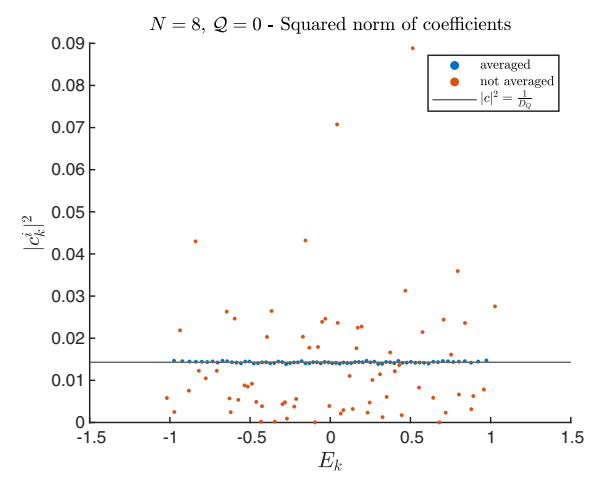

(a)

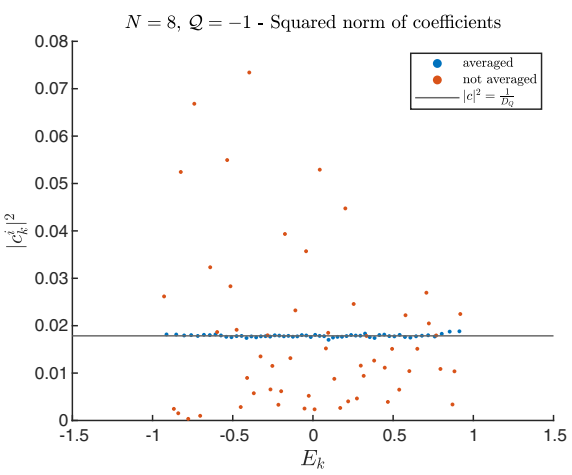

(b)

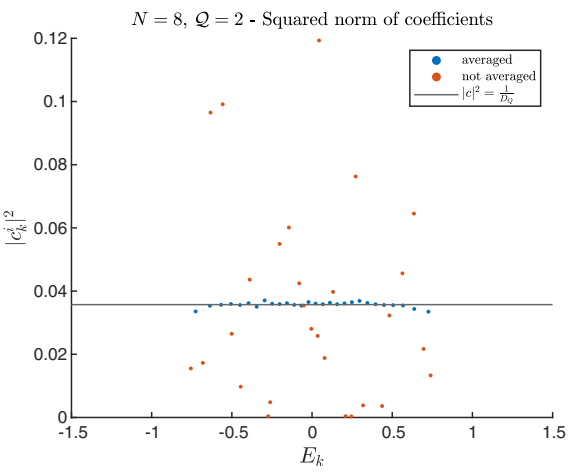

(c)

FIG. 8. Squared norms of coefficients. $\left|c_{k}^{i}\right|^{2}$ for a given Fock state $\left|S_{i}\right\rangle$ and for every energy eigenstate $\left|E_{k}\right\rangle$ in the same charge subsector. The red points are the squared norms for a single realization of the Hamiltonian, while the blue points are averaged over 5000 realizations. The mean of the squared norm is clearly $1 / D_{\mathcal{Q}}$ (horizontal line). (a) $\mathcal{Q}=0$. (b) $\mathcal{Q}=-1$. (c) $\mathcal{Q}=2$.

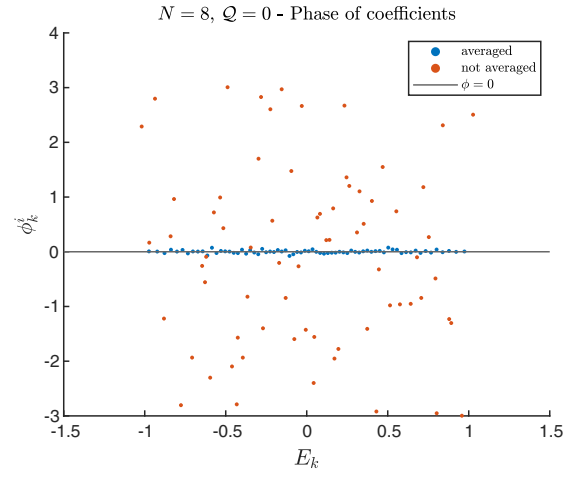

(a)

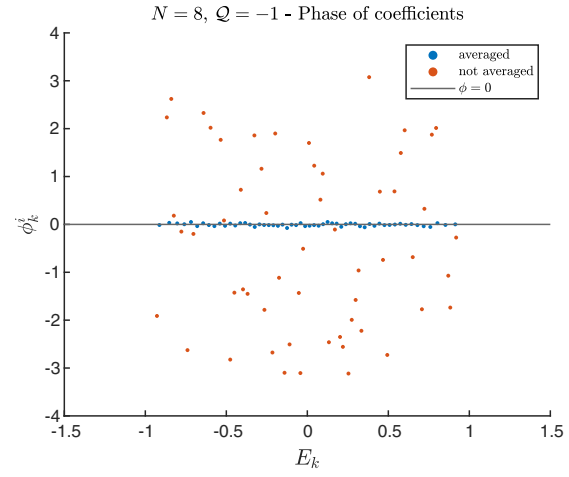

(b)

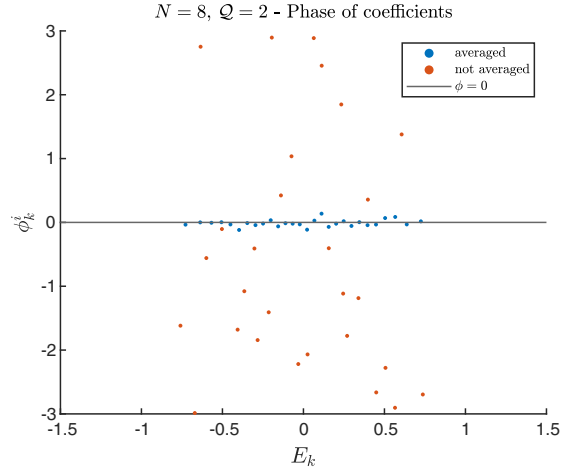

(c)

FIG. 9. Phases of coefficients. Phases of $c_{k}^{i}$ for a given Fock state $\left|S_{i}\right\rangle$ and for every energy eigenstate $\left|E_{k}\right\rangle$ in the same charge subsector. The red points are the phases for a single realization of the Hamiltonian, while the blue points are averaged over 5000 realizations. The phases are clearly uniformly distributed between $-\pi$ and $\pi$, and therefore their mean vanishes. (a) $\mathcal{Q}=0$. (b) $\mathcal{Q}=-1$. (c) $\mathcal{Q}=2$.

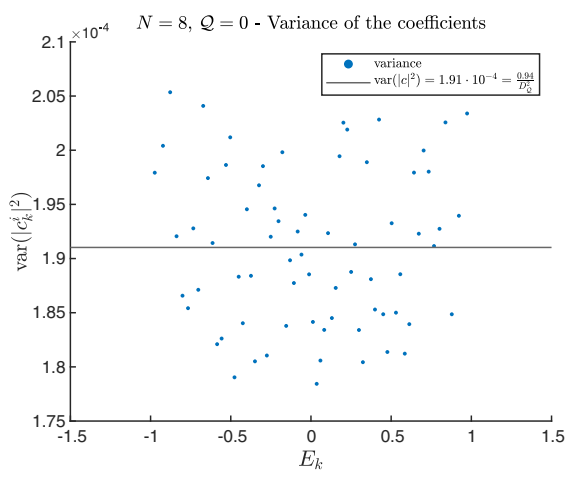

(a)

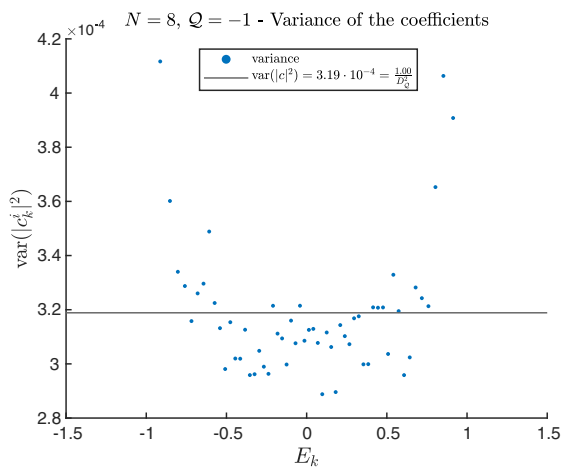

(b)

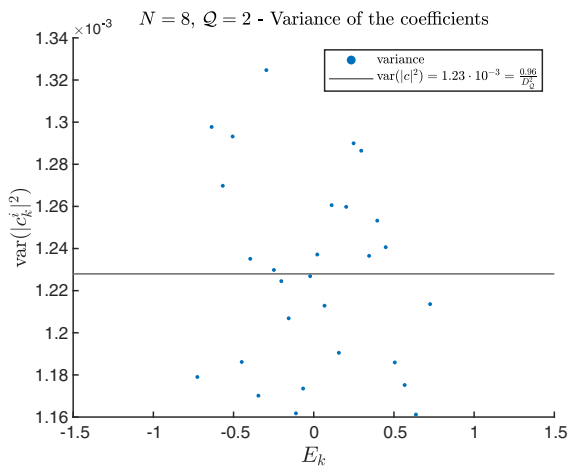

(c)

FIG. 10. Variance of the squared norms of coefficients. Variance of $\left|c_{k}^{i}\right|^{2}$ for a given Fock state $\left|S_{i}\right\rangle$ and for every energy eigenstate $\left|E_{k}\right\rangle$ in the same charge subsector, averaged over 5000 realizations. The variance is of order $1 / D_{\mathcal{Q}}^{2}$ (horizontal line). (a) $\mathcal{Q}=0$. (b) $\mathcal{Q}=-1$. (c) $\mathcal{Q}=2$. 


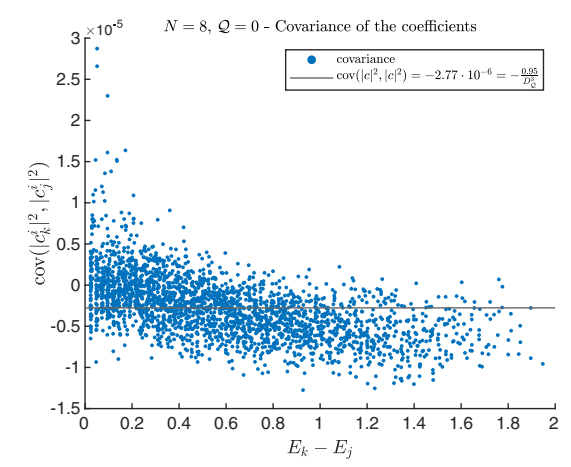

(a)

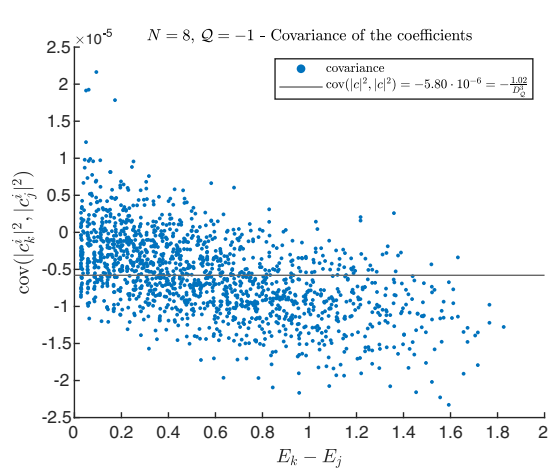

(b)

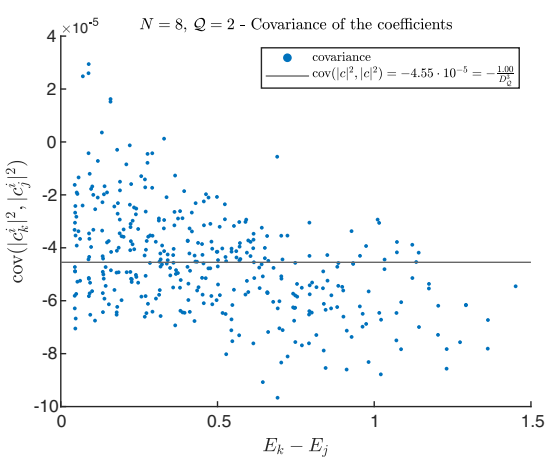

(c)

FIG. 11. Covariance of the squared norms of coefficients. Covariance of $\left|c_{k}^{i}\right|^{2}$ and $\left|c_{j}^{i}\right|^{2}$ for a given Fock state $\left|S_{i}\right\rangle$ and for all energy eigenstates $\left|E_{k}\right\rangle$ and $\left|E_{j}\right\rangle$ in the same charge subsector, averaged over 5000 realizations. The covariance is of order $1 / D_{\mathcal{Q}}^{3}$ (horizontal line). (a) $\mathcal{Q}=0$. (b) $\mathcal{Q}=-1$. (c) $\mathcal{Q}=2$.

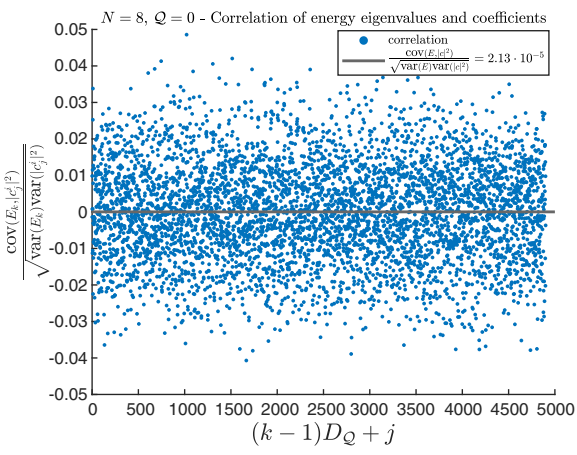

(a)

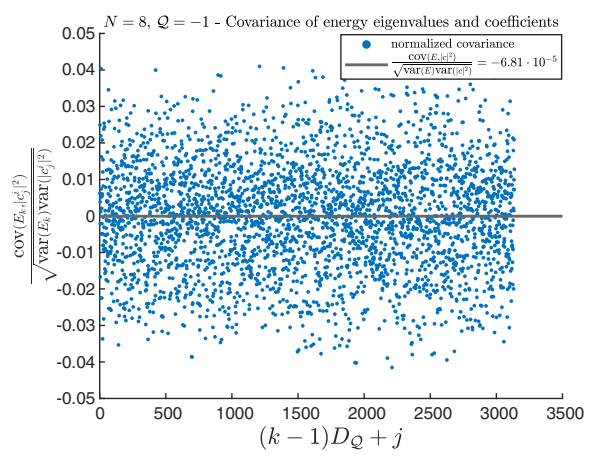

(b)

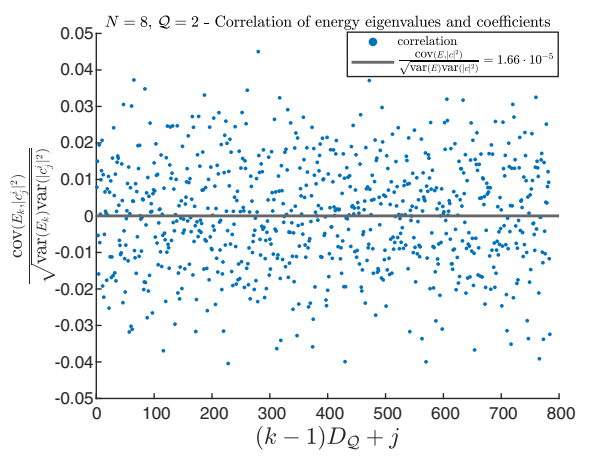

(c)

FIG. 12. Correlation between squared norms of coefficients and energy eigenvalues. Correlation between $\left|c_{j}^{i}\right|^{2}$ and energy eigenvalues $E_{k}$ for a given Fock state $\left|S_{i}\right\rangle$ and for all energy eigenstates $\left|E_{j}\right\rangle$ and $\left|E_{k}\right\rangle$ in the same charge subsector, averaged over 5000 realizations. The correlation is clearly strongly suppressed. (a) $\mathcal{Q}=0$. (b) $\mathcal{Q}=-1$. (c) $\mathcal{Q}=2$.

\section{Two-point functions}

In Sec. III B we showed that the denominator of Eq. (15) is given by Eq. (17). Let us now focus on the numerator of Eq. (15). Since we are inserting a specific fermion $\hat{\psi}_{k}$, if $\tau_{0}-\left|\tau_{1,2}\right|$ is not large enough to end up in a generic superposition of all the Fock states in the charge subsector before the insertion of the fermion, the numerator of Eq. (15) depends on the occupation number of the $k$ th fermion. However, given the properties of the coefficients $c_{j}^{i}$ outlined in Sec. III A and Appendix B 1, it is still independent of the occupation number of all the other $N-1$ fermions after averaging over disorder.

Given a charge subsector with charge $\mathcal{Q}$, there are $n_{\uparrow, \mathcal{Q}}=\mathcal{Q}+N / 2$ fermions in the $|\uparrow\rangle$ state. Then the $D_{\mathcal{Q}}=$ $\left(\begin{array}{c}N \\ n_{\uparrow, Q}\end{array}\right)$ states in the Fock basis of the charge subsector are split up in $D_{\mathcal{Q}, k=\uparrow}=\left(\begin{array}{c}N-1 \\ \left(n_{\uparrow, \mathcal{Q}}\right)-1\end{array}\right)$ states with the $k$ th fermion in the $|\uparrow\rangle$ state and $D_{\mathcal{Q}, k=\downarrow}=\left(\begin{array}{c}N-1 \\ n_{\uparrow, \mathcal{Q}}\end{array}\right)$ states with the $k$ th fermion in the $|\downarrow\rangle$ state.
We can therefore consider the numerator of Eq. (15) and sum over all the $D_{\mathcal{Q}, k=\uparrow}$ (correspondingly, $D_{\mathcal{Q}, k=\downarrow}$ ) Fock states with the $k$ th fermion in the $|\uparrow\rangle$ (correspondingly, $|\downarrow\rangle$ ) state. This is equivalent to summing over all the $D_{\mathcal{Q}}$ Fock states in the charge subsector after inserting the projector $P_{k=\uparrow}=\hat{\psi}_{k}^{\dagger}\left(-\tau_{0}\right) \hat{\psi}_{k}\left(-\tau_{0}\right)$ [correspondingly, $P_{k=\downarrow}=\hat{\psi}_{k}\left(-\tau_{0}\right)$ $\left.\hat{\psi}_{k}^{\dagger}\left(-\tau_{0}\right)\right]$. Using the cyclic property of the trace we obtain

$$
\begin{aligned}
& \sum_{\left\{\left|S_{i, k=\uparrow}\right\rangle\right\}}\left\langle S_{i}\left|\mathrm{e}^{-\tau_{0} \hat{H}} T\left[\hat{\psi}_{k}\left(\tau_{1}\right) \hat{\psi}_{k}^{\dagger}\left(\tau_{2}\right)\right] \mathrm{e}^{-\tau_{0} \hat{H}}\right| S_{i}\right\rangle \\
& \quad=\operatorname{Tr}_{\mathcal{Q}}\left(\mathrm{e}^{-2 \tau_{0} \hat{H}} T\left[\hat{\psi}_{k}\left(\tau_{1}\right) \hat{\psi}_{k}^{\dagger}\left(\tau_{2}\right) \hat{\psi}_{k}^{\dagger}\left(-\tau_{0}+0^{+}\right) \hat{\psi}_{k}\left(-\tau_{0}\right)\right]\right),
\end{aligned}
$$

$$
\begin{aligned}
& \sum_{\left\{\left|S_{i, k=\downarrow}\right\rangle\right\}}\left\langle S_{i}\left|\mathrm{e}^{-\tau_{0} \hat{H}} T\left[\hat{\psi}_{k}\left(\tau_{1}\right) \hat{\psi}_{k}^{\dagger}\left(\tau_{2}\right)\right] \mathrm{e}^{-\tau_{0} \hat{H}}\right| S_{i}\right\rangle \\
& \quad=\operatorname{Tr}_{\mathcal{Q}}\left(\mathrm{e}^{-2 \tau_{0} \hat{H}} T\left[\hat{\psi}_{k}\left(\tau_{1}\right) \hat{\psi}_{k}^{\dagger}\left(\tau_{2}\right) \hat{\psi}_{k}\left(-\tau_{0}+0^{+}\right) \hat{\psi}_{k}^{\dagger}\left(-\tau_{0}\right)\right]\right) .
\end{aligned}
$$


After averaging over disorder, each element in the sum on the left-hand sides does not depend on the occupation number of the remaining $N-1$ fermions, and has the same occupation number for the $k$ th fermion: all the elements of the sum take the same value. Since the distribution of the coefficients is sharply peaked, at leading order in $1 / D_{\mathcal{Q}}$ the latter property is still valid for a single drawing of the couplings. This is the same self-averaging property invoked in Sec. III B for the denominator of Eq. (15). Therefore we obtain

$$
\begin{aligned}
& \left\langle S_{i, k=\uparrow}\left|\mathrm{e}^{-\tau_{0} \hat{H}} T\left[\hat{\psi}_{k}\left(\tau_{1}\right) \hat{\psi}_{k}^{\dagger}\left(\tau_{2}\right)\right] \mathrm{e}^{-\tau_{0} \hat{H}}\right| S_{i, k=\uparrow}\right\rangle=\frac{1}{D_{\mathcal{Q}, k=\uparrow}} \operatorname{Tr}_{\mathcal{Q}}\left(\mathrm{e}^{-2 \tau_{0} \hat{H}} T\left[\hat{\psi}_{k}\left(\tau_{1}\right) \hat{\psi}_{k}^{\dagger}\left(\tau_{2}\right) \hat{\psi}_{k}^{\dagger}\left(-\tau_{0}+0^{+}\right) \hat{\psi}_{k}\left(-\tau_{0}\right)\right]\right), \\
& \left\langle S_{i, k=\downarrow}\left|\mathrm{e}^{-\tau_{0} \hat{H}} T\left[\hat{\psi}_{k}\left(\tau_{1}\right) \hat{\psi}_{k}^{\dagger}\left(\tau_{2}\right)\right] \mathrm{e}^{-\tau_{0} \hat{H}}\right| S_{i, k=\downarrow}\right\rangle=\frac{1}{D_{\mathcal{Q}, k=\downarrow}} \operatorname{Tr}_{\mathcal{Q}}\left(\mathrm{e}^{-2 \tau_{0} \hat{H}} T\left[\hat{\psi}_{k}\left(\tau_{1}\right) \hat{\psi}_{k}^{\dagger}\left(\tau_{2}\right) \hat{\psi}_{k}\left(-\tau_{0}+0^{+}\right) \hat{\psi}_{k}^{\dagger}\left(-\tau_{0}\right)\right]\right) .
\end{aligned}
$$

Note that these expectation values are of order $D_{\mathcal{Q}}^{0}$. Finally, dividing Eqs. (B4) and (B5) by Eq. (17), at leading order in $1 / D_{\mathcal{Q}}$ we find the result reported in Eq. (18).

\section{a. Numerical results}

We report here for completeness additional numerical results for the two-point functions. In particular, the singlefermion and collective two-point functions in the $\mathcal{Q}=-2$ charge subsector for $N=8, \tau_{0}=50$ are plotted in Fig. 13 for a single realization of the Hamiltonian and in Fig. 16 averaged over 200 realizations of the Hamiltonian. Note that, as we have pointed out in footnote 6 , when evolving for a long amount of Euclidean time we end up in the ground state of the corresponding charge subsector if $N<$ $\beta J$ like in this case. In particular, in Figs. 13 and 16 we insert $\hat{\psi}^{\dagger}$ at time $\tau_{2}=0$ and $\hat{\psi}$ at time $\tau_{1}$. Therefore, when $\tau_{1} \lesssim \tau_{0}$, at time $\tau=0$ the bra is roughly the ground state of the $\mathcal{Q}=-1$ charge subsector. We then insert $\hat{\psi}^{\dagger}$ and take the scalar product with the ket (which is roughly the ground state of the $\mathcal{Q}=-2$ charge subsector), obtaining in the numerator a result of order $\exp \left[-\tau_{0}\left(E_{-1}+E_{-2}\right)\right]$, where $E_{-1}$ and $E_{-2}$ are the energy eigenvalues of the ground states of the $\mathcal{Q}=-1$ and $\mathcal{Q}=-2$ charge subsectors, respectively. The normalization factor will clearly be of order $\exp \left[-2 \tau_{0} E_{-2}\right]$. Therefore we obtain a result of order $\exp \left[-\tau_{0}\left(E_{-1}-E_{-2}\right)\right]$. The plots in Figs. 13 and 16 suggest a spacing $\left|E_{-2}-E_{-1}\right|=\mathcal{O}\left(10^{-1}\right)$ and $E_{-1}<E_{-2}$, in accordance with our numerical result and [44]. On the other hand, for $\tau_{1} \gtrsim-\tau_{0}$, we expect a result of order $\exp \left[-\tau_{0}\left(E_{-3}-E_{-2}\right)\right]$, which is exponentially suppressed

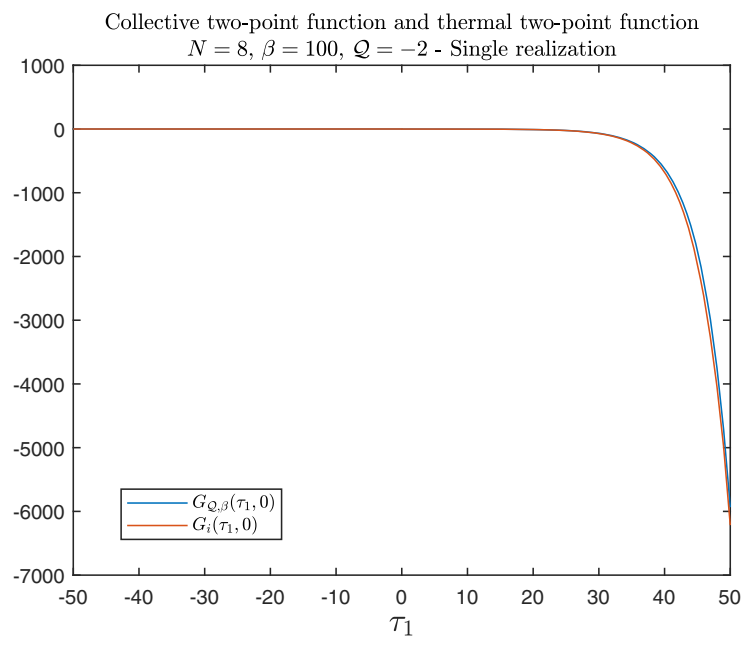

(b)

FIG. 13. Single-fermion and collective two-point functions. $\mathcal{Q}=-2, N=8, k=1, \beta=2 \tau_{0}=100$, single realization of the Hamiltonian. The expectations outlined in Secs. III B and III C are matched to good accuracy. The evident asymmetry is expected and explained in Appendix B 2. (a) Single-fermion two-point functions. The left-hand sides and right-hand sides of Eq. (18) are plotted along with the thermal two-point function in the corresponding charge subsector. (b) Collective two-point functions. The left-hand side and right-hand side of Eq. (23) are plotted. 


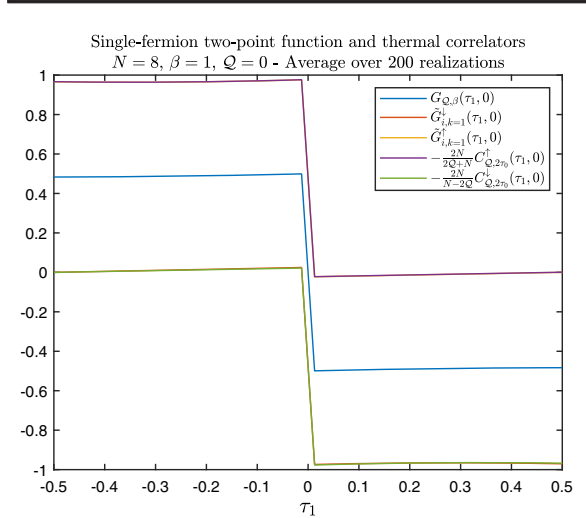

(a)

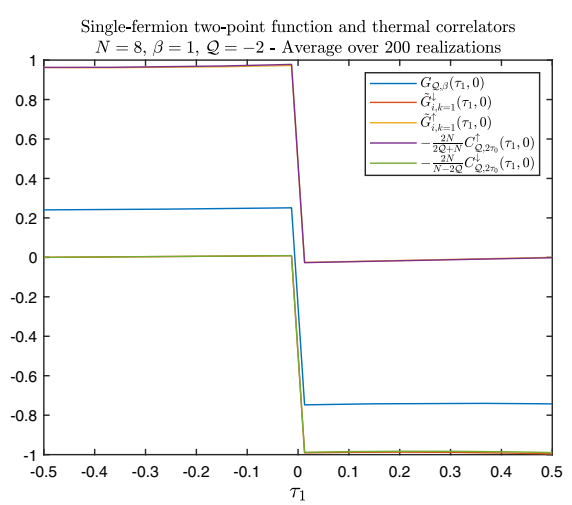

(b)

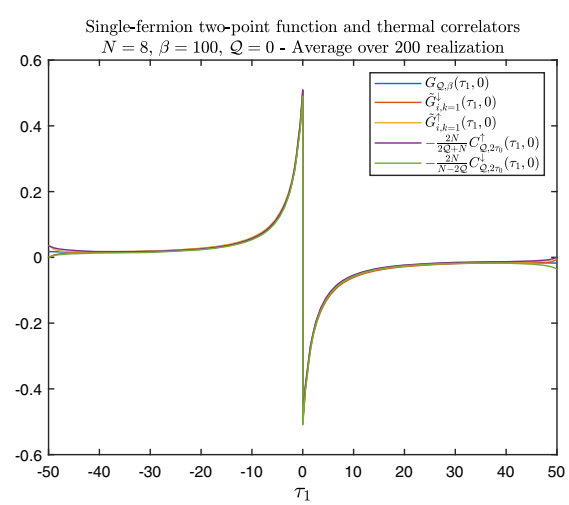

(c)

FIG. 14. Single-fermion two-point functions: average over disorder. The left-hand sides and right-hand sides of Eq. (18) are plotted along with the thermal two-point function in the corresponding charge subsector for $N=8$ and $k=1$, averaged over 200 realizations of the Hamiltonian. The expectations outlined in Sec. III B are matched to good accuracy, and more precisely than for a single realization of the Hamiltonian, as expected. (a) $\mathcal{Q}=0, \beta=2 \tau_{0}=1$. (b) $\mathcal{Q}=-2, \beta=2 \tau_{0}=1$. (c) $\mathcal{Q}=0, \beta=2 \tau_{0}=100$.

since $E_{-3}>E_{-2}$, hence the asymmetry observed in Figs. 13 and 16. For the $\mathcal{Q}=0$ charge subsector (see Figs. 1, 2, 14, and 15), this effect is much smaller because $\left|E_{0}-E_{ \pm 1}\right|$ is about one order of magnitude smaller than $\left|E_{-2}-E_{-1}\right|$. Note also that the behavior of the singlefermion two-point function (and the corresponding thermal four-point function) in Figs. 13 and 16 when inserting the fermion very close to $\tau=\tau_{0}$ depends on the specific boundary state chosen. In particular, when $\hat{\psi}_{1}$ is inserted right next to the bra Fock state (i.e., at $\tau=\tau_{0}$ ) with the first fermion in the $|\uparrow\rangle$ state, the state is annihilated and the correlator correctly vanishes.

Finally, we also report in Figs. 14 and 15 the same plots shown in Figs. 1 and 2, but averaged over 200 realizations of the Hamiltonian. As expected, the accuracy of the result (18), which is already consistent for a single realization of the Hamiltonian, is increased when averaging over disorder.

\section{Expectation values of generic operators}

In Sec. III D we claimed that under appropriate assumptions, the expectation value $\left\langle B_{i}|\hat{O}| B_{i}\right\rangle$ of a generic operator $\hat{O}$ over a boundary state is equivalent to the thermal expectation value computed in the same charge subsector. In particular, given the properties of the probability distribution of the coefficients $c_{i}^{k}$, such statement is valid at leading order in $1 / D_{\mathcal{Q}}$ both after averaging over disorder and for a single realization of the Hamiltonian. Let us now prove this result, working under the assumptions listed in Sec. III D.

First, let us clarify assumption 2, concerning the set of operators for which the claim holds. As a simple example, consider an operator of the form

$$
\hat{O}=T\left[\prod_{i \in M^{-}} \hat{\psi}_{i}\left(\tau_{i}^{-}\right) \prod_{j \in M^{+}} \hat{\psi}_{j}^{\dagger}\left(\tau_{j}^{+}\right)\right]
$$

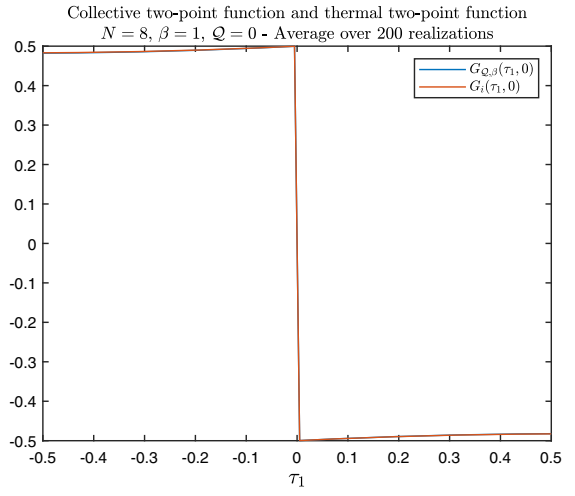

(a)

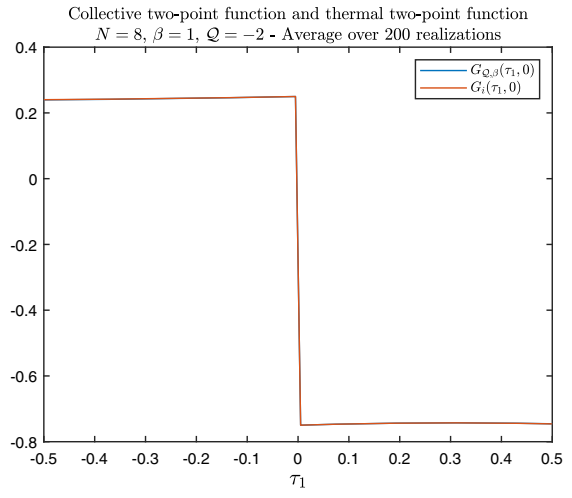

(b)

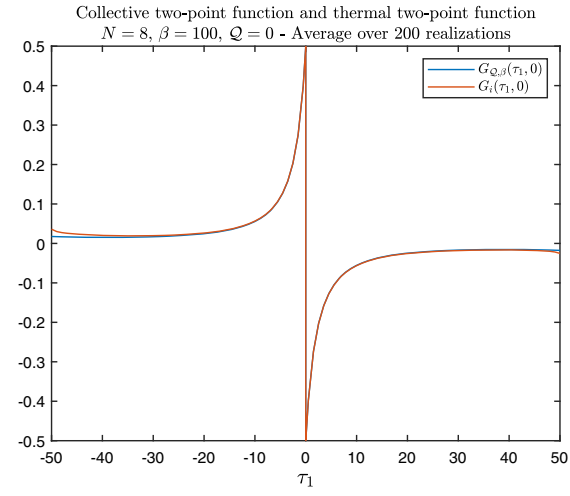

(c)

FIG. 15. Collective two-point functions: average over disorder. The left-hand side and right-hand side of Eq. (23) are plotted for $N=8$ and $k=1$, averaged over 200 realizations of the Hamiltonian. The expectations outlined in Sec. III C are matched to good accuracy, and more precisely than for a single realization of the Hamiltonian, as expected. (a) $\mathcal{Q}=0, \beta=2 \tau_{0}=1$. (b) $\mathcal{Q}=-2, \beta=2 \tau_{0}=1$. (c) $\mathcal{Q}=0, \beta=2 \tau_{0}=100$. 


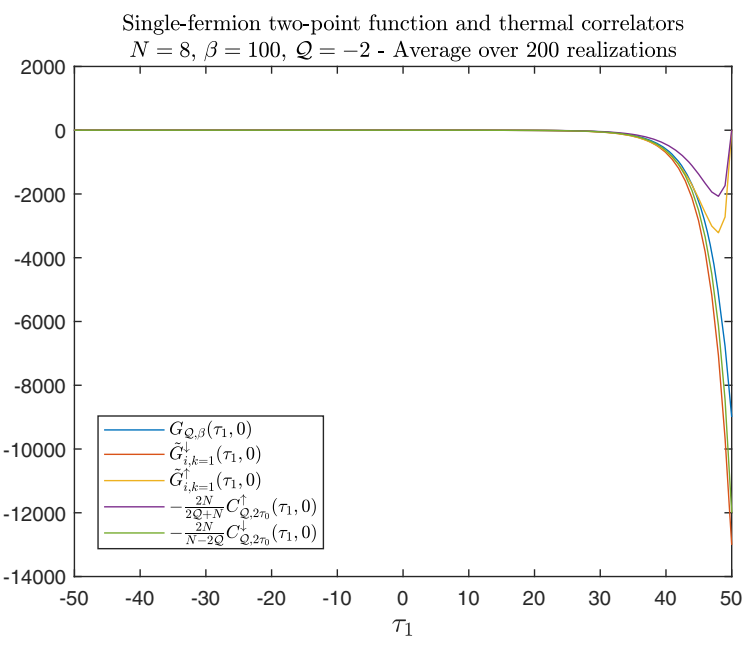

(a)

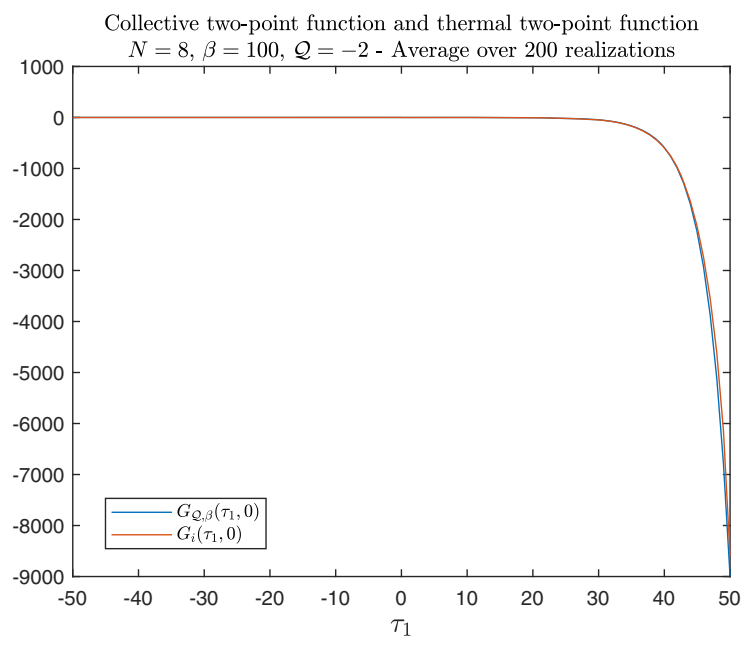

(b)

FIG. 16. Single-fermion and collective two-point functions: average over disorder. $\mathcal{Q}=-2, N=8, k=1, \beta=2 \tau_{0}=100$, average over 200 realizations of the Hamiltonian. The expectations outlined in Secs. III B and III C are matched to good accuracy, and more precisely than for a single realization of the Hamiltonian. The evident asymmetry is expected and explained in Appendix B 2. (a) Singlefermion two-point functions. The left-hand sides and right-hand sides of Eq. (18) are plotted along with the thermal two-point function in the corresponding charge subsector. (b) Collective two-point functions. The left-hand side and right-hand side of Eq. (23) are plotted.

where $M^{+}$and $M^{-}$are index sets of equal size (the case of nonequal size is trivial since operators with unequal numbers of creation and annihilation operators have zero expected value in a fixed-charge subsector). Provided the number of insertions is much less than $N$ and provided all the times $\tau_{i}^{ \pm}$are well separated from $\pm \tau_{0}$, we expect such operators to have thermal-looking expectation values over a boundary state, up to corrections suppressed by $1 / N$ and $\exp \left(-\left(\tau_{0}-\left|\tau_{i}^{ \pm}\right|\right) J\right)$. Naturally, this can be the case only if the preparation time is large enough, i.e., $\tau_{0} J \gg 1$.

A simple example where assumption 2 fails is when $\hat{O}=\mathrm{e}^{\tau_{0} \hat{H}} \hat{\psi}_{i}^{\dagger} \hat{\psi}_{i} \mathrm{e}^{-\tau_{0} \hat{H}}$. In this case, the fermion occupation number operator is inserted right next to the Fock state and hence is sensitive to the precise occupation number of the $i$ th fermion. However, collective fields like

$$
\hat{O}_{\text {coll }}=T\left[\frac{1}{N} \sum_{i=1}^{N} \hat{\psi}_{i}^{\dagger}\left(\tau_{1}\right) \hat{\psi}_{i}\left(\tau_{2}\right)\right]
$$

are better behaved. Note that the operator (B7) is invariant under permutations of the fermions. The Hamiltonian evolution is also effectively permutation invariant at large $N$ due to the emergent $U(N)$ symmetry. It follows that the expectation value of permutation-invariant collective operators such as (B7) is independent of the specific boundary state up to $1 / N$-suppressed corrections, regardless of the value of the preparation time $\tau_{0}$ and of the location of the fermion insertion points. The collective two-point functions studied in Sec. IIIC provide a clear example of this behavior.
Given a generic operator $\hat{O}$ satisfying assumptions 2 and 3, we define $\alpha_{i j} \equiv\left\langle E_{i}|\hat{O}| E_{j}\right\rangle \exp \left(-\tau_{0}\left(E_{i}+E_{j}\right)\right)$ and $\beta_{i} \equiv \exp \left(-2 \tau_{0} E_{i}\right)$. Using Eq. (12), we can then write the expectation value over a boundary state $\left|B_{i}\right\rangle$ as

$$
\left\langle O_{i}\right\rangle \equiv\left\langle B_{i}|\hat{O}| B_{i}\right\rangle=\frac{\sum_{k, l=1}^{D_{\mathcal{Q}}}\left|c_{k}^{i}\right|\left|c_{l}^{i}\right| \alpha_{l k} \mathrm{e}^{i\left(\theta_{k}-\theta_{l}\right)}}{\sum_{k=1}^{D_{\mathcal{Q}}}\left|c_{k}^{i}\right|^{2} \beta_{k}},
$$

where $\theta_{k}$ are the phases of the coefficients $c_{k}^{i}$. Averaging over the phases, which are uniformly distributed, we get

$$
{\overline{\langle O\rangle_{i}}}_{i}=\frac{\sum_{k=1}^{D_{\mathcal{Q}}}\left|c_{k}^{i}\right|^{2} \alpha_{k k}}{\sum_{k=1}^{D_{\mathcal{Q}}}\left|c_{k}^{i}\right|^{2} \beta_{k}} .
$$

(This statement fails for $\hat{O}=\mathrm{e}^{\tau_{0} \hat{H}} \hat{\psi}_{i}^{\dagger} \hat{\psi}_{i} \mathrm{e}^{-\tau_{0} \hat{H}}$ because the $\alpha_{l k}$ can be correlated with the phases.) We will show at the end of the present section that the average over the squared norms of the coefficients can be taken separately in the numerator and denominator, and the average of the ratio is equal to the ratio of the averages up to corrections of order $1 / D_{\mathcal{Q}}$. Therefore, we obtain immediately ${ }^{15}$

$$
\overline{\overline{\langle O\rangle}}_{i}=\frac{\sum_{k=1}^{D_{\mathcal{Q}}} \alpha_{k k}}{\sum_{k=1}^{D_{\mathcal{Q}}} \beta_{k}}+\mathcal{O}\left(\frac{1}{D_{\mathcal{Q}}}\right),
$$

which is the result (26) (a single overline indicates an average over the phases and a double overline an average

\footnotetext{
${ }^{15} \mathrm{~A}$ similar result for the expectation value of the Hamiltonian in analogous cSYK pure states was found in [53].
} 
over both the phases and the squared norms of the coefficients). Therefore, averaging over disorder, generic correlators over a boundary state are equal, up to corrections suppressed at large $N$, to the thermal correlators in the same charge subsector. Note that when averaging over the coefficients we used assumption 1 to ignore the (small) correlation between the coefficients and the energy eigenvalues contained in $\alpha_{i j}$ and $\beta_{i}$.

Let us now analyze the size of fluctuations around the average value (B10). Taking the square of Eq. (B8) and averaging over the phases we obtain (all the sums are from 1 to $\left.D_{\mathcal{Q}}\right)$

$\overline{\langle O\rangle_{i}^{2}}=\frac{\sum_{k \neq l}\left|c_{k}^{i}\right|^{2}\left|c_{l}^{i}\right|^{2}\left(\alpha_{k k} \alpha_{l l}+\alpha_{l k} \alpha_{k l}\right)+\sum_{k}\left|c_{k}^{i}\right|^{4} \alpha_{k k}^{2}}{\sum_{k, l}\left|c_{k}^{i}\right|^{2}\left|c_{l}^{i}\right|^{2} \beta_{k} \beta_{l}}$.

Again, at leading order in $1 / D_{\mathcal{Q}}$ the average over the squared norms can be taken separately in the numerator and denominator. Subtracting the square of Eq. (B10) and keeping only the leading orders we obtain the following expression for the variance of the expectation value:

$$
\begin{aligned}
& \overline{\overline{\langle O\rangle_{i}^{2}}}-{\overline{\overline{\langle O\rangle_{i}}}}_{i} \\
& =\frac{\sum_{k \neq l, n} \alpha_{l k} \alpha_{k l} \beta_{m} \beta_{n}+a \sum_{k, l, m}\left(\alpha_{k k}^{2} \beta_{l} \beta_{m}-\alpha_{k k} \alpha_{l l} \beta_{m}^{2}\right)}{\left(\sum_{k, l} \beta_{k} \beta_{l}\right)^{2}} \\
& \quad+\mathcal{O}\left(\frac{1}{D_{\mathcal{Q}}}\right),
\end{aligned}
$$

where $a \approx 1$ is the variance of the norm of the coefficients. Let us now evaluate the order of each term in Eq. (B12). For a generic $\tau_{0}, \beta_{k}$ and $\alpha_{k k}$ are $\mathcal{O}(1)$, while $\alpha_{k l}=\mathcal{O}\left(1 / \sqrt{D_{\mathcal{Q}}}\right)$ for $k \neq l$ by assumption 3. The first sum in the numerator contains $D_{\mathcal{Q}}^{3}\left(D_{\mathcal{Q}}-1\right) \approx D_{\mathcal{Q}}^{4}$ terms, the second sum in the numerator contains $D_{\mathcal{Q}}^{3}$ terms, and the sum in the denominator contains $D_{\mathcal{Q}}^{4}$ terms. Therefore we obtain

$$
\overline{\overline{\langle O\rangle_{i}^{2}}}-{\overline{\overline{\langle O\rangle_{i}}}}_{i}=\mathcal{O}\left(\frac{1}{D_{\mathcal{Q}}}\right)
$$

which is Eq. (27). Note that if we exclude the subsectors with maximal and minimal charge, $D_{\mathcal{Q}} \geq N$. Since the size of the fluctuations of a generic correlator around its disorder-averaged value is controlled by the square root of the variance (B13), it is suppressed in the large $N$ limit. This implies that also for a single realization of the Hamiltonian the expectation value of a generic operator $\hat{O}$ over a boundary state approaches in the large $N$ limit the thermal expectation value in the same charge subsector computed by the canonical ensemble at fixed charge. In other words, boundary states look thermal. This result generalizes the analytic and numerical results for the twopoint functions analyzed in Secs. III B and IIIC and in Appendix B 2.

In order to obtain the results (B10) and (B13) we averaged the numerator and denominator separately over the norms of the coefficients, and then took the ratio of the averages. To complete our proof we need to show that, at leading order in $1 / D_{\mathcal{Q}}$, this procedure is equivalent to taking the average of the ratio. ${ }^{16}$ Let us denote by $\overline{\mathcal{N}}$ and $\overline{\mathcal{D}}$ the numerator and denominator of Eqs. (B9) or (B11). Then we can write $\overline{\mathcal{N}}=\overline{\overline{\mathcal{N}}}+\overline{\delta \mathcal{N}}$ and $\overline{\mathcal{D}}=\overline{\overline{\mathcal{D}}}+\overline{\delta \mathcal{D}}$. Both $\overline{\overline{\mathcal{N}}}$ and $\overline{\overline{\mathcal{D}}}$ are $\mathcal{O}(1)$ numbers. By construction $\overline{\overline{\delta \mathcal{N}}}=\overline{\overline{\delta \mathcal{D}}}=0$. Using the properties of the coefficients $c_{k}^{i}$, the size of the fluctuations of $\overline{\delta \mathcal{N}}$ and $\overline{\delta \mathcal{D}}$ is controlled by $\left(\overline{\overline{\mathcal{N}^{2}}}-\overline{\overline{\mathcal{N}}}^{2}\right)^{\frac{1}{2}}=\mathcal{O}\left(D_{\mathcal{Q}}^{-\frac{1}{2}}\right)$ and $\left(\overline{\overline{\mathcal{D}^{2}}}-\overline{\overline{\mathcal{D}}}^{2}\right)^{\frac{1}{2}}=\mathcal{O}\left(D_{\mathcal{Q}}^{-\frac{1}{2}}\right)$, respectively. Therefore, in the large $N$ limit $\delta \overline{\mathcal{N}} / \overline{\overline{\mathcal{N}}} \ll 1$ and $\delta \overline{\mathcal{D}} / \overline{\overline{\mathcal{D}}} \ll 1$ with high probability. In the same way, it is possible to show that $\overline{\delta \overline{\mathcal{N}} \delta \overline{\mathcal{D}}}=\overline{\overline{(\mathcal{N D})}}-(\overline{\overline{\mathcal{N}}})(\overline{\overline{\mathcal{D}}})=\mathcal{O}\left(D_{\mathcal{Q}}^{-1}\right) . \quad$ We $\quad$ can $\quad$ then write

$$
\overline{\left(\frac{\mathcal{N}}{\mathcal{D}}\right)}=\frac{\overline{\mathcal{N}}}{\overline{\mathcal{D}}}=\frac{\overline{\overline{\mathcal{N}}}}{\overline{\overline{\mathcal{D}}}}\left(1+\frac{\delta \overline{\mathcal{N}}}{\overline{\overline{\mathcal{N}}}}-\frac{\delta \overline{\mathcal{D}}}{\overline{\overline{\mathcal{D}}}}+\frac{\delta \overline{\mathcal{N}} \delta \overline{\mathcal{D}}}{(\overline{\overline{\mathcal{N}}})(\overline{\overline{\mathcal{D}}})}\right)+\mathcal{O}\left(\frac{1}{D_{\mathcal{Q}}}\right),
$$

which immediately implies

$$
\overline{\overline{\left(\frac{\mathcal{N}}{\mathcal{D}}\right)}}=\frac{\overline{\bar{N}}}{\overline{\bar{D}}}+\mathcal{O}\left(\frac{1}{D_{\mathcal{Q}}}\right)
$$

which is the desired result.

\section{APPENDIX C: DIMENSIONAL REDUCTION OF BRANEWORLD COSMOLOGY}

We will give here additional details regarding the dimensional reduction of the braneworld cosmology action $S=$ $S_{\text {bulk }}+S_{\text {ETw }}$ proposed in [11]. We will follow mainly the conventions of $[31,33]$ (see also $[25,26,32,63]$ ), to which we refer for additional details and discussion. The action is the one for a $(d+1)$-dimensional Einstein-Maxwell theory at fixed charge with a negative cosmological constant and a dynamical brane with constant tension $T_{\text {ETw: }}$

\footnotetext{
${ }^{16}$ Note that this property holds exactly for the average over the phases.
} 


$$
\begin{aligned}
S_{\mathrm{bulk}} & =-\frac{1}{16 \pi G} \int_{\mathcal{M}^{(d+1)}} d^{d+1} x \sqrt{g_{(d+1)}}\left(R_{(d+1)}-2 \Lambda-4 \pi G F_{\mu \nu}^{(d+1)} F_{(d+1)}^{\mu \nu}\right)+S_{\mathrm{GHY}}+S_{\infty}^{e m,} \\
S_{\mathrm{ETW}} & =-\frac{1}{8 \pi G} \int_{\mathrm{ETW}^{(d)}} d^{d} y \sqrt{h_{d}}\left[K_{d}^{\mathrm{ETW}}-(d-1) T_{\mathrm{ETW}}\right]+S_{\mathrm{ETW}}^{e m},
\end{aligned}
$$

where $\mathcal{M}^{(d+1)}$ indicates the spacetime manifold, $g_{(d+1)}$ is the determinant of the metric, $R_{(d+1)}$ is the Ricci scalar, $\Lambda$ is the negative cosmological constant [given by $\Lambda=-d(d-1)$ / $\left.\left(2 L_{\text {AdS }}^{2}\right)\right], F_{\mu \nu}^{(d+1)}$ is the electromagnetic tensor, $h_{d}$ is the determinant of the metric induced on the brane, and $K_{d}^{\mathrm{ETW}}$ is the trace of the extrinsic curvature of the brane. We used $y^{A}$ (with $A=0, \ldots, d-1$ ) to denote the coordinates on the brane, and we will use the same notation for the asymptotic boundary. Greek indices $\mu, \nu$ run instead from 0 to $d$. $S_{\mathrm{GHY}}$ is the Gibbons-Hawking-York boundary term, given by

$$
S_{\mathrm{GHY}}=-\frac{1}{8 \pi G} \int_{\partial \mathcal{M}_{\infty}^{(d)}} d^{d} y \sqrt{\gamma_{d}} K_{d}^{\infty},
$$

with $\partial \mathcal{M}_{\infty}^{(d)}$ denoting the asymptotic boundary, $\gamma_{d}$ the induced metric on it, and $K_{d}^{\infty}$ the trace of its extrinsic curvature. The electromagnetic boundary terms $S_{\infty}^{e m}$ and $S_{\text {ETW }}^{e m}$ are needed to have a well-posed variational problem when we hold the charge fixed [54]. Their expression is (we report here only the asymptotic one, but the ETW term is completely analogous)

$$
S_{\infty}^{e m}=-\int_{\partial \mathcal{M}_{\infty}^{(d)}} d^{d} y \sqrt{\gamma_{d}} F_{(d+1)}^{\mu \nu} n_{\mu}^{\infty} A_{\nu}
$$

where $n_{\mu}^{\infty}$ is the one-form dual to the unit vector normal to the asymptotic boundary and $A_{\mu}$ is the electromagnetic potential.

For the sake of dimensional reduction, it is useful to introduce the following ansatz for the spacetime metric, including a Weyl rescaling of the two-dimensional metric:

$$
d s^{2}=\frac{r_{e}^{d-2}}{\phi^{d-2}} g_{i j} d x^{i} d x^{j}+\phi^{2}\left(x^{i}\right) d \Omega_{d-1}^{2}
$$

where $g_{i j}$ is a two-dimensional metric and $\phi\left(x^{i}\right)$ is the dilaton, which is a generic function of the two-dimensional coordinates only (indicated by lower case latin indices $i$, $j=0,1)$. Using the ansatz (C4) in the braneworld cosmology action and integrating out the angular part we obtain the following dimensionally reduced action:

$$
\begin{aligned}
S= & -\frac{V_{d-1}}{16 \pi G} \int_{\mathcal{M}} d^{2} x \sqrt{g}\left[\phi^{d-1} R+U(\phi)-4 \pi G Z(\phi) F_{i j} F^{i j}\right]-\frac{V_{d-1}}{8 \pi G} \int_{\partial \mathcal{M}_{\infty}} d u \sqrt{\gamma_{u u}}\left[\phi^{d-1} K^{\infty}+8 \pi G Z(\phi) F^{i j} n_{i}^{\infty} A_{j}\right] \\
& -\frac{V_{d-1}}{8 \pi G} \int_{\mathrm{ETW}} d v \sqrt{h_{v v}}\left[\phi^{d-1} K^{\mathrm{ETW}}-(d-1) \phi^{\frac{d}{2}} r_{e}^{\frac{d}{2}-1} T_{\mathrm{ETW}}+8 \pi G Z(\phi) F^{i j} n_{i}^{\mathrm{ETW}} A_{j}\right],
\end{aligned}
$$

where we defined

$$
\begin{aligned}
& U(\phi)=r_{e}^{d-2}\left[\frac{d(d-1)}{L_{\mathrm{AdS}}^{2}} \phi+\frac{(d-1)(d-2)}{\phi}\right], \\
& Z(\phi)=\frac{\phi^{2 d-3}}{r_{e}^{d-2}} .
\end{aligned}
$$

As we have already discussed, we are not interested in charge fluctuations, and therefore we can integrate out the gauge field in the path integral [32]. This sums up to solving Maxwell's equations and computing the bulk and boundary electromagnetic terms on shell. After long but straightforward calculations, we obtain the effective action

$$
\begin{aligned}
S_{\text {eff }}= & -\frac{V_{d-1}}{16 \pi G} \int_{\mathcal{M}} d^{2} x \sqrt{g}\left[\phi^{d-1} R+U(\phi)-(d-1)(d-2) \frac{r_{e}^{d-2}}{\phi^{2 d-3}} Q^{2}\right]-\frac{V_{d-1}}{8 \pi G} \int_{\partial \mathcal{M}_{\infty}} d u \sqrt{\gamma_{u u}} \phi^{d-1} K^{\infty} \\
& -\frac{V_{d-1}}{8 \pi G} \int_{\mathrm{ETW}} d v \sqrt{h_{v v}}\left[\phi^{d-1} K^{\mathrm{ETW}}-(d-1) \phi^{\frac{d}{2} r_{e}^{\frac{d}{2}-1}} T_{\mathrm{ETW}}\right],
\end{aligned}
$$


where the charge parameter $Q$ is uniquely determined by the extremal horizon radius $r_{e}$ (or vice versa) [11].

The dimensionally reduced effective action (C7) is valid all the way to the asymptotic boundary and for any value of the charge parameter $Q$. In order to obtain the JT gravity action, we must take the near-extremal, near-horizon limit of Eq. (C7) and regularize the asymptotic boundary to the region specified by Eq. (41). Note that on shell the dilaton $\phi$ plays the role of the radius of the $(d-1)$-dimensional sphere $S^{d-1}$. Therefore, in the extremal limit and at the black hole horizon $\phi=\phi_{0}=r_{e}$, and in the near-extremal, near-horizon limit it will vary slightly from this value.
It is useful for our purposes to redefine the dilaton as $\Phi=\phi^{d-1}$. We can then split the redefined dilaton in a constant piece $\Phi_{0}=r_{e}^{d-1}$ and a dynamical field $\Phi_{1}$, i.e., $\Phi=\Phi_{0}+\Phi_{1}$. In the near-extremal, near-horizon limit $\Phi_{1} \ll \Phi_{0}$ holds, and the on-shell expression for $\Phi_{1}$ (which will be useful to derive the ETW particle trajectory in Appendix D) at leading order in $R_{2}^{2} / r_{e} z$ is

$$
\Phi_{1} \approx(d-1) r_{e}^{d-2} \frac{R_{2}^{2}}{z} .
$$

We can now expand the effective action (C7) to first order in $\Phi_{1} / \Phi_{0}$, finally obtaining the result (42), where

$$
\begin{aligned}
S_{0} & =-\frac{V_{d-1} \Phi_{0}}{16 \pi G}\left[\int_{\mathcal{M}} d^{2} x \sqrt{g} R+2 \int_{\partial \mathcal{M}_{\infty}} d u \sqrt{\gamma_{u u}} K^{\infty}+2 \int_{\mathrm{ETW}} d v \sqrt{h_{v v}} K^{\mathrm{ETW}}\right], \\
S_{0}^{\mathrm{ETW}} & =\frac{(d-1) V_{d-1} \Phi_{0}^{\frac{d}{2(d-1)}} T_{\mathrm{ETW}}}{8 \pi G} \int_{\mathrm{ETW}} d v \sqrt{h_{v v}} .
\end{aligned}
$$

$S_{0}$ is a topological term and it is a constant by GaussBonnet theorem. ${ }^{17} S_{0}^{\mathrm{ETW}}$ is proportional to the proper length of the ETW particle trajectory, and will give a contribution to the equations of motion of the ETW particle, as discussed in Appendix D.

\section{APPENDIX D: SCHWARZIAN ACTION AND ETW PARTICLE TRAJECTORY}

In this Appendix we will outline the steps needed to obtain the Schwarzian effective action (47) governing the gravitational dynamics in the near-horizon region of the near-extremal black hole, and derive the NN boundary conditions that must be imposed at the location of the ETW particle, leading to the trajectory described in Sec. V C.

As we have already pointed out, we must integrate out the dilaton $\Phi_{1}$ and compute the effective action (42) on shell. The only nonvanishing and nonconstant term is the GHY term for the regularized boundary. The metric is fixed to be the two-dimensional part of Eq. (40), and the induced metric and the dilaton on the regularized boundary must satisfy the DD boundary conditions (43). After parametrizing the trajectory of the regularized boundary in the bulk by $(\tau(u), z(u))$, the boundary condition for the metric leads to Eq. (46), while the one-form dual to the outward-pointing unit vector normal to the regularized boundary is given by $n_{i}^{\infty}=N\left(z^{\prime}(u),-\tau^{\prime}(u)\right)$, where $N$ is an appropriate normalization factor that can be computed by imposing $g^{i j} n_{i}^{\infty} n_{j}^{\infty}=1$. At second order in $\varepsilon$ the extrinsic curvature then reads

\footnotetext{
${ }^{17} \mathrm{We}$ remind that the boundary of the manifold $\mathcal{M}$ is $\partial \mathcal{M}=\partial \mathcal{M}_{\infty} \cup E T W$.
}

$$
K^{\infty}=\frac{1}{R_{2}}+\varepsilon^{2} R_{2}\{\tan (\pi T \tau(u)), u\}+\mathcal{O}\left(\varepsilon^{4}\right)
$$

Using again the DD boundary conditions (43), the GHY term for the regularized boundary takes the form

$$
I_{\mathrm{GHY}} \approx-\frac{V_{d-1} \phi_{b}}{8 \pi G} \int_{\partial \mathcal{M}_{\infty}} d u\left[\frac{1}{R_{2} \varepsilon^{2}}+R_{2}\{\tan (\pi T \tau(u)), u\}\right] .
$$

Note that the first term is divergent for $\varepsilon \rightarrow 0$. In order to cancel such constant divergent term, generally in JT gravity a counterterm

$$
I_{c t}=\frac{V_{d-1}}{8 \pi G} \int_{\partial \mathcal{M}_{\infty}} d u \sqrt{\gamma_{u u}} \Phi_{1} \frac{1}{R_{2}}
$$

is introduced in the action. We could do the same here. However, since we are working at small but finite cutoff $\varepsilon$, the first term in Eq. (D2) is a constant finite term that does not pose particular problems and does not contribute to the dynamics, so the introduction of the counterterm is not necessary. In either case, we can discard such term together with any other constant term in the on-shell action, obtaining the effective action (47). Note that in the effective action $u \in[-\beta / 2, \beta / 2]$ if the ETW particle does not intersect the regularized boundary, while $u \in\left[-u_{0}, u_{0}\right]$ if the ETW particle intersects the regularized boundary at $u= \pm u_{0}$. In the tangent case of our interest, $u_{0}=\beta / 2$ and therefore the integral in Eq. (47) runs over the whole thermal circle, i.e., $u \in[-\beta / 2, \beta / 2]$.

Let us now focus on the ETW particle action. As we have mentioned, we want to impose NN boundary conditions at 


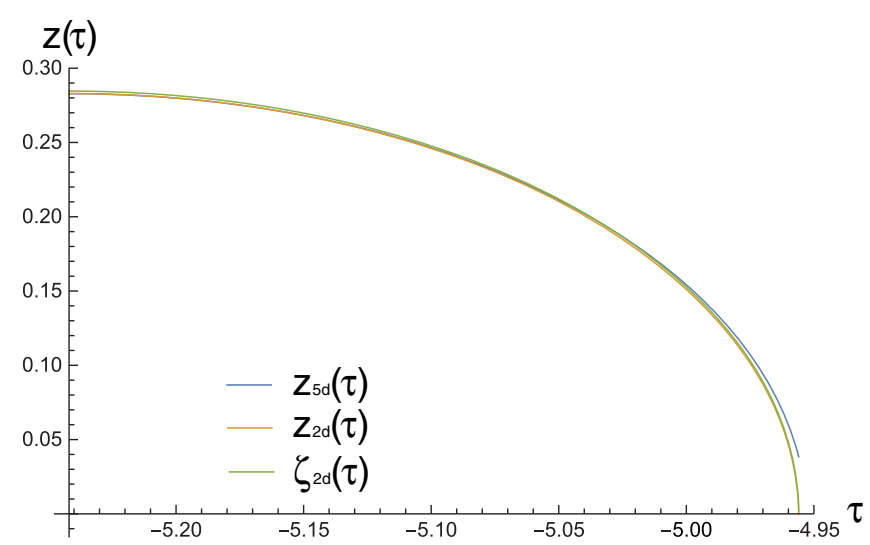

(a)

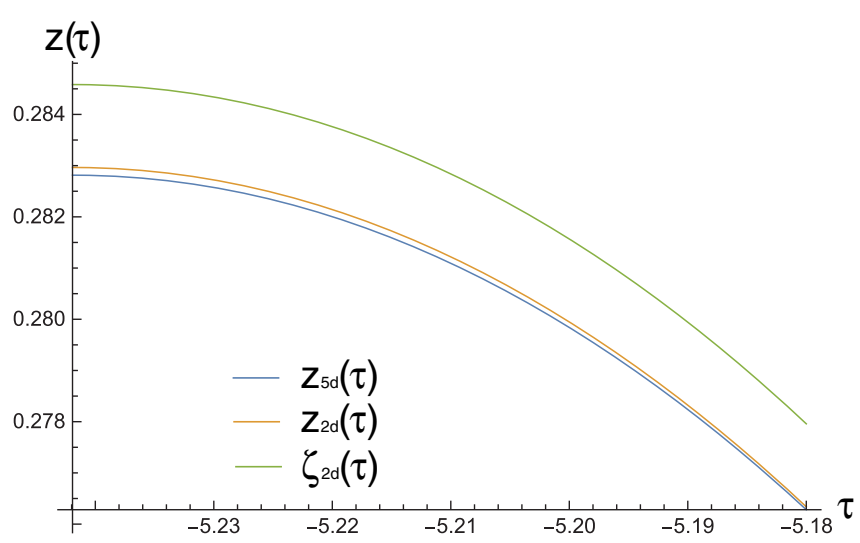

(b)

FIG. 17. ETW particle and brane trajectories: expansion phase. $d=4, L_{\mathrm{AdS}}=1, r_{+}=100, r_{-}=99.9, T_{\mathrm{ETW}}=0.01$. The black hole is near extremal and the tension is low. The brane enters the near-horizon region $\left(z(\tau) \gg R_{2}^{2} / r_{-} \gtrsim R_{2}^{2} / r_{e}\right)$. The exact numerical solution $\zeta_{2 d}(\tau)$ of Eq. (48) and the approximate analytic solution $z_{2 d}(\tau)$ [given by Eq. (51)] are in good agreement with each other, and with the numerical solution $z_{5 d}(\tau)$ of the higher-dimensional ETW brane equation of motion [11]. The agreement is particularly good in the nearhorizon region (larger $z$ ), where our dimensionally reduced analysis is sensible, as expected. (a) Expansion phase between $z=$ $z_{\max } \approx 0.28$ and the asymptotic boundary at $z=0$. (b) Detail of the near-horizon region.

the location of the ETW particle. This implies that when the effective action (42) is varied, the variations $\delta \sqrt{h_{v v}}$ and $\left.\delta \Phi_{1}\right|_{\text {etw }}$ do not vanish. The total ETW particle term in the variation of the action (42) is given by the sum of the variation of the ETW particle action and boundary terms arising from the variation of the bulk action ${ }^{18}$ [65]:

$$
\begin{aligned}
\delta S_{\mathrm{tot}}^{E T W}= & -\frac{V_{d-1}}{8 \pi G} \int_{E T W} d v\left(\left\{n_{E T W}^{i} \partial_{i} \Phi_{1}-\Phi_{1} K^{E T W}-(d-1) \Phi_{0}^{\frac{d}{2(d-1)}} T_{E T W}\right.\right. \\
& \left.\left.+\Phi_{1}\left[K^{E T W}-\frac{d}{2} \Phi_{0}^{-\frac{d-2}{2(d-1)}} T_{E T W}\right]\right\} \delta\left(\sqrt{h_{v v}}\right)+\sqrt{h_{v v}}\left[K^{E T W}-\frac{d}{2} \Phi_{0}^{-\frac{d-2}{2(d-1)}} T_{E T W}\right] \delta \Phi_{1}\right) .
\end{aligned}
$$

The NN boundary conditions we are looking for are obtained by imposing $\delta S_{\text {tot }}^{E T W}=0$, which immediately leads to the boundary conditions (44) and (45).

The ETW particle trajectory can be obtained from Eq. (45). It is useful to parametrize the ETW particle trajectory using the bulk time $\tau$ [i.e., $v=\tau, z=z(\tau)$ ], which implies $n_{i}^{E T W}=\tilde{N}\left(z^{\prime}(\tau),-1\right)$, where again $\tilde{N}$ is a normalization factor. By substituting the expression for the outward-pointing normal unit vector $n_{E T W}^{i}=g^{i j} n_{j}^{E T W}$ and the on-shell expression for the dilaton (C8) into Eq. (45), we obtain the ETW particle equation of motion (48).

Equation (48) cannot be solved analytically. However, the expression of the inversion point can be found explicitly by requiring the square root to vanish:

$$
z_{\max }=\frac{d r_{e} R_{2}^{2} T_{E T W}^{2}}{2\left(r_{e}^{2} T_{E T W}^{2}+4 \pi^{2} T^{2} R_{2}^{2}\right)}\left[-1+\sqrt{1+\frac{\left(r_{e}^{2} T_{E T W}^{2}+4 \pi^{2} T^{2} R_{2}^{2}\right)\left(4-d^{2} R_{2}^{2} T_{E T W}^{2}\right)}{d^{2} R_{2}^{2} r_{e}^{2} T_{E T W}^{4}}}\right] .
$$

Note that $z_{\max } \rightarrow 1 /(2 \pi T)$ as $T_{E T W} \rightarrow 0$, i.e., the minimum radius of the ETW particle is the black hole horizon radius, which is in agreement with the higher-dimensional result [11].

In the near-horizon region of our interest, the condition $z \gg R_{2}^{2} / r_{e}$ holds. Since we are only interested in the case where the ETW particle crosses the regularized boundary and enters the near-horizon region, we can use the condition $z(\tau) \gg$ $R_{2}^{2} / r_{e}$ to rewrite Eq. (48) as

\footnotetext{
${ }^{18}$ Note that the last term of the first line arises from the variation of $S_{0}^{E T W}$.
} 
$z^{\prime}(\tau) \approx \pm R_{2} \frac{1-4 \pi^{2} T^{2} z^{2}}{T_{E T W} r_{e} z} \sqrt{1-4 \pi^{2} T^{2} z^{2}-\frac{r_{e}^{2} T_{E T W}^{2}}{R_{2}^{2}} z^{2}}$.

Note that we could not drop the $(2 \pi T z)^{2}$ terms, because even if they are small in the region where the regularized boundary sits [determined by Eq. (41)] they are of order 1 when the ETW particle is very close to the horizon, i.e., when $z(\tau) \gtrsim 1 /(2 \pi T)$. This approximate equation of motion does have an analytic solution, which is given by Eq. (51).
We finally report in Fig. 17 a plot of the expansion phase of the ETW particle trajectory for low tension in a near-extremal black hole for $d=4$, obtained from the approximate analytic solution (51) [indicated by $z_{2 d}(\tau)$ ] and by solving numerically Eq. (48) [indicated by $\zeta_{2 d}(\tau)$ ]. The trajectory of the ETW brane obtained by solving numerically the brane equation of motion in the full higher-dimensional model [11] is also plotted [indicated by $z_{5 d}(\tau)$ ). We remark how the trajectories are all in good agreement with each other, and particularly so in the near-horizon region where our dimensionally reduced two-dimensional effective action is sensible and approximately equivalent to the full five-dimensional braneworld cosmology action.
[1] J. M. Maldacena, The large N limit of superconformal field theories and supergravity, Adv. Theor. Math. Phys. 2, 231 (1998).

[2] E. Witten, Anti-de Sitter space and holography, Adv. Theor. Math. Phys. 2, 253 (1998).

[3] S. S. Gubser, I. R. Klebanov, and A. M. Polyakov, Gauge theory correlators from noncritical string theory, Phys. Lett. B 428, 105 (1998).

[4] O. Aharony, S. S. Gubser, J. M. Maldacena, H. Ooguri, and Y. Oz, Large $\mathrm{N}$ field theories, string theory and gravity, Phys. Rep. 323, 183 (2000).

[5] G. 't Hooft, Dimensional reduction in quantum gravity, in Conference on Highlights of Particle and Condensed Matter Physics (SALAMFEST) Trieste, Italy, 1993; Conf. Proc. C 930308, 284 (1993) [arXiv:gr-qc/9310026].

[6] L. Susskind, The World as a hologram, J. Math. Phys. (N.Y.) 36, 6377 (1995).

[7] T. Takayanagi, Holographic Dual of BCFT, Phys. Rev. Lett. 107, 101602 (2011).

[8] M. Fujita, T. Takayanagi, and E. Tonni, Aspects of AdS/ BCFT, J. High Energy Phys. 11 (2011) 043.

[9] A. Karch and L. Randall, Open and closed string interpretation of SUSY CFT's on branes with boundaries, J. High Energy Phys. 06 (2001) 063.

[10] S. Cooper, M. Rozali, B. Swingle, M. Van Raamsdonk, C. Waddell, and D. Wakeham, Black hole microstate cosmology, J. High Energy Phys. 07 (2019) 065.

[11] S. Antonini and B. Swingle, Cosmology at the end of the world, Nat. Phys. 16, 881 (2020).

[12] L. Randall and R. Sundrum, Large Mass Hierarchy from a Small Extra Dimension, Phys. Rev. Lett. 83, 3370 (1999).

[13] L. Randall and R. Sundrum, An Alternative to Compactification, Phys. Rev. Lett. 83, 4690 (1999).

[14] A. Karch and L. Randall, Locally localized gravity, J. High Energy Phys. 05 (2001) 008.

[15] M. Van Raamsdonk, Cosmology from confinement?, arXiv: 2102.05057.
[16] A. Almheiri, T. Hartman, J. Maldacena, E. Shaghoulian, and A. Tajdini, Replica wormholes and the entropy of hawking radiation, J. High Energy Phys. 05 (2020) 013.

[17] G. Penington, S. H. Shenker, D. Stanford, and Z. Yang, Replica wormholes and the black hole interior, arXiv: 1911.11977.

[18] Y. Li, X. Chen, and M. P. A. Fisher, Quantum Zeno effect and the many-body entanglement transition, Phys. Rev. B 98, 205136 (2018).

[19] B. Skinner, J. Ruhman, and A. Nahum, MeasurementInduced Phase Transitions in the Dynamics of Entanglement, Phys. Rev. X 9, 031009 (2019).

[20] A. Chan, R. M. Nandkishore, M. Pretko, and G. Smith, Unitary-projective entanglement dynamics, Phys. Rev. B 99, 224307 (2019).

[21] I. Kourkoulou and J. Maldacena, Pure states in the SYK model and nearly-AdS $S_{2}$ gravity, arXiv:1707.02325.

[22] A. Almheiri, A. Mousatov, and M. Shyani, Escaping the interiors of pure boundary-state black holes, arXiv:1803.04434.

[23] M. Van Raamsdonk, Comments on wormholes, ensembles, and cosmology, arXiv:2008.02259.

[24] J. Maldacena and D. Stanford, Remarks on the Sachdev-YeKitaev model, Phys. Rev. D 94, 106002 (2016).

[25] J. Maldacena, D. Stanford, and Z. Yang, Conformal symmetry and its breaking in two dimensional nearly Anti-de-Sitter space, Prog. Theor. Exp. Phys. 2016, 12C104 (2016).

[26] G. Sárosi, $\mathrm{AdS}_{2}$ holography and the SYK model, Proc. Sci., Modave2017 (2018) 001 [arXiv:1711.08482].

[27] R. Jackiw, Lower dimensional gravity, Nucl. Phys. B252, 343 (1985).

[28] C. Teitelboim, Gravitation and Hamiltonian structure in two space-time dimensions, Phys. Lett. 126B, 41 (1983).

[29] S. Sachdev and J. Ye, Gapless Spin Fluid Ground State in a Random, Quantum Heisenberg Magnet, Phys. Rev. Lett. 70, 3339 (1993).

[30] A. Kitaev, A simple model of quantum holography, http:// online.kitp.ucsb.edu/online/entangled15/ (2015), kITP strings seminar and Entanglement 2015 program. 
[31] S. Sachdev, Universal low temperature theory of charged black holes with $\mathrm{AdS}_{2}$ horizons, J. Math. Phys. (N.Y.) 60, 052303 (2019).

[32] A. R. Brown, H. Gharibyan, H. W. Lin, L. Susskind, L. Thorlacius, and Y. Zhao, Complexity of Jackiw-Teitelboim gravity, Phys. Rev. D 99, 046016 (2019).

[33] P. Nayak, A. Shukla, R. M. Soni, S. P. Trivedi, and V. Vishal, On the dynamics of near-extremal black holes, J. High Energy Phys. 09 (2018) 048.

[34] U. Moitra, S. P. Trivedi, and V. Vishal, Extremal and nearextremal black holes and near-CFT 1 , J. High Energy Phys. 07 (2019) 055.

[35] A. Gaikwad, L. K. Joshi, G. Mandal, and S. R. Wadia, Holographic dual to charged SYK from 3D gravity and Chern-Simons, J. High Energy Phys. 02 (2020) 033.

[36] P. Saad, S. H. Shenker, and D. Stanford, A semiclassical ramp in SYK and in gravity, arXiv:1806.06840.

[37] P. Saad, S. H. Shenker, and D. Stanford, JT gravity as a matrix integral, arXiv:1903.11115.

[38] C. V. Johnson, Nonperturbative Jackiw-Teitelboim gravity, Phys. Rev. D 101, 106023 (2020).

[39] C. V. Johnson, Explorations of non-perturbative JT gravity and supergravity, Phys. Rev. D 103, 046013 (2021).

[40] Y. Gu, X.-L. Qi, and D. Stanford, Local criticality, diffusion and chaos in generalized Sachdev-Ye-Kitaev models, J. High Energy Phys. 05 (2017) 125.

[41] F. Ferrari and F. I. Schaposnik Massolo, Phases of melonic quantum mechanics, Phys. Rev. D 100, 026007 (2019).

[42] S. Sahoo, E. Lantagne-Hurtubise, S. Plugge, and M. Franz, Traversable wormhole and Hawking-Page transition in coupled complex SYK models, Phys. Rev. Research 2, 043049 (2020).

[43] S. Sachdev, Bekenstein-Hawking Entropy and Strange Metals, Phys. Rev. X 5, 041025 (2015).

[44] Y. Gu, A. Kitaev, S. Sachdev, and G. Tarnopolsky, Notes on the complex Sachdev-Ye-Kitaev model, J. High Energy Phys. 02 (2020) 157.

[45] P. Chaturvedi, Y. Gu, W. Song, and B. Yu, A note on the complex SYK model and warped CFTs, J. High Energy Phys. 12 (2018) 101.

[46] J. Liu and Y. Zhou, Note on global symmetry and SYK model, J. High Energy Phys. 05 (2019) 099.

[47] R. A. Davison, W. Fu, A. Georges, Y. Gu, K. Jensen, and S. Sachdev, Thermoelectric transport in disordered metals without quasiparticles: The Sachdev-Ye-Kitaev models and holography, Phys. Rev. B 95, 155131 (2017).
[48] D. Stanford and E. Witten, JT gravity and the ensembles of random matrix theory, arXiv:1907.03363.

[49] P. Coleman, Introduction to Many-Body Physics (Cambridge University Press, Cambridge, England, 2015), https://doi.org/10.1017/CBO9781139020916.

[50] Y. D. Lensky, X.-L. Qi, and P. Zhang, Size of bulk fermions in the SYK model, J. High Energy Phys. 10 (2020) 053.

[51] M. Srednicki, Chaos and quantum thermalization, Phys. Rev. E 50, 888 (1994).

[52] J. M. Deutsch, Quantum statistical mechanics in a closed system, Phys. Rev. A 43, 2046 (1991).

[53] J. Sonner and M. Vielma, Eigenstate thermalization in the Sachdev-Ye-Kitaev model, J. High Energy Phys. 11 (2017) 149.

[54] S. Hawking and S. F. Ross, Duality between electric and magnetic black holes, Phys. Rev. D 52, 5865 (1995).

[55] A. Chamblin, R. Emparan, C. V. Johnson, and R. C. Myers, Charged AdS black holes and catastrophic holography, Phys. Rev. D 60, 064018 (1999).

[56] J. L. Hovdebo and R. C. Myers, Bouncing brane worlds go crunch!, J. Cosmol. Astropart. Phys. 11 (2003) 012.

[57] S. S. Seahra, C. Clarkson, and R. Maartens, Delocalization of brane gravity by a bulk black hole, Classical Quant. Grav. 22, L91 (2005).

[58] S. Ryu and T. Takayanagi, Holographic Derivation of Entanglement Entropy from AdS/CFT, Phys. Rev. Lett. 96, 181602 (2006).

[59] V.E. Hubeny, M. Rangamani, and T. Takayanagi, A covariant holographic entanglement entropy proposal, J. High Energy Phys. 07 (2007) 062.

[60] A. Almheiri, R. Mahajan, J. Maldacena, and Y. Zhao, The Page curve of Hawking radiation from semiclassical geometry, J. High Energy Phys. 03 (2020) 149.

[61] A. Almheiri, R. Mahajan, and J. Maldacena, Islands outside the horizon, arXiv:1910.11077.

[62] G. Penington, Entanglement Wedge reconstruction and the information paradox, J. High Energy Phys. 09 (2020) 002.

[63] U. Moitra, S. K. Sake, S. P. Trivedi, and V. Vishal, JackiwTeitelboim gravity and rotating black holes, J. High Energy Phys. 11 (2019) 047.

[64] P. Gao, D. L. Jafferis, and D. K. Kolchmeyer, An effective matrix model for dynamical end of the world branes in Jackiw-Teitelboim gravity, arXiv:2104.01184.

[65] A. Goel, L. V. Iliesiu, J. Kruthoff, and Z. Yang, Classifying boundary conditions in JT gravity: From energy-branes to $\alpha$-branes, arXiv:2010.12592. 